DOI: 10.1002/adom.((please add manuscript number))

Submitted to

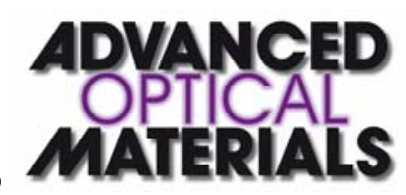

Article type: Review

\title{
Ultrafast Photophysics of Single-Walled Carbon Nanotubes
}

Giancarlo Soavi*, Francesco Scotognella, Guglielmo Lanzani and Giulio Cerullo*

Dr. Giancarlo Soavi, Dr. Francesco Scotognella, Prof. Giulio Cerullo

Dipartimento di Fisica, Politecnico di Milano, piazza Leonardo da Vinci 32, 20133, Milano, Italy

e-mail: gs544@cam.ac.uk; giulio.cerullo@polimi.it

Prof. Guglielmo Lanzani

Center for Nano Science and Technology@PoliMi, Istituto Italiano di Tecnologia, Via Giovanni Pascoli, 70/3, 20133 Milano

Keywords: carbon nanotubes; excited state dynamics; excitons; ultrafast spectroscopy

\begin{abstract}
Single-walled carbon nanotubes (SWNTs) are nano-cylinders obtained by wrapping one layer of graphene; due to their very high aspect ratio, they are the prototypical quantum confined one-dimensional systems. The unique mechanical, electronic and optical properties of SWNTs open up transversal application possibilities in many fields of science and technology, with particular emphasis on optoelectronics and photonics. A prerequisite for many of these applications is a thorough understanding of the nature and dynamics of their elementary excitations. This review aims at summarizing the current understanding of the ultrafast photophysics of SWNTs, based on two decades of experimental investigations. After discussing the morphological and electronic properties of SWNTs and introducing the different photogenerated species, we will briefly describe the ultrafast spectroscopic techniques most commonly used for their characterization. We will then present the experimental evidence that has led to establish the nature (singlet and triplet excitons, biexcitons, trions and free charges) and the relaxation pathways of photoexcitations in SWNTs.
\end{abstract}

\section{Introduction}

The discovery of Carbon Nanotubes (CNTs) is matter of debate.[1] Indeed, CNTs have been reported at least three times, in 1952, 1976 and then 1991.[2][3][4] Only in the XXI 
Submitted to

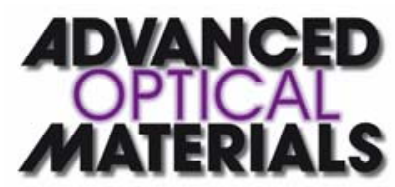

century, however, CNTs have aroused a broad fundamental and technological interest, possibly due to the widespread awareness about nanotechnology. CNTs have unique physical properties that potentially suggest a broad range of applications in mechanics, microelectronics, bio-medicine, optoelectronics and photonics.[5][6][7][8][9][10][11][12] CNTs are continuous cylinders obtained by wrapping one (single-walled, SWNT) or more (multi-walled, MWNT) layers of graphene, with open or close ends.[4][11] In analogy with other carbon allotropes, such as fullerenes and graphene, in CNTs the carbon-carbon bonds arise from the overlap of both the $\mathrm{sp}^{2}$ orbitals, i.e. the strong $\sigma$ bonds in the $\mathrm{x}-\mathrm{y}$ plane, and the $2 \mathrm{p}_{\mathrm{z}}$ orbitals, i.e. the weak $\pi$ bonds in the $\mathrm{z}$ direction. The $\sigma$ bonds are responsible for the structural (mechanical) properties of the material, while the $\pi$ bonds govern their electronic properties. Over more than two decades of intense research, CNT production techniques have evolved from electrical discharge with graphite electrodes[4] to pulsed laser vaporization[13][14] and chemical vapour deposition.[15][16][17] Dispersion of CNTs in solution can be achieved with the use of surfactants or specific solvents and disparate sorting and separation strategies have been proposed.[18][19][20][21][22][23][24]

There is a large crowd of CNTs. They have diameters ranging from 0.8 to $2 \mathrm{~nm}$ for SWNTs, and from 5 to more than $100 \mathrm{~nm}$ for MWNTs, and their length varies from hundreds of $\mathrm{nm}$ to centimetres [8] with a consequent very high aspect ratio, making them an excellent approximation of a one-dimensional (1D) quantum-confined solid. MWNTs are usually metallic and can transport currents up to $10^{9} \mathrm{~A} / \mathrm{cm}^{2}$.[25] Instead, SWNTs can be either metallic or semiconducting, according to the orientation of the graphene sheet with respect to the tube axis (i.e. the tube chirality). CNTs have outstanding mechanical properties, with elastic modulus approaching $1 \mathrm{TPa}$ and a tensile strength of $100 \mathrm{GPa}$ measured for an individual MWNT.[26] Moreover, individual SWNTs can have a very high thermal conductivity $(3500 \mathrm{~W} / \mathrm{m} \cdot \mathrm{K})$ at room temperature, exceeding that of diamond, depending on the wall area.[27]

Nowadays CNTs, either pure or embedded in composite materials, are employed in a variety of innovative devices with enhanced mechanical, electronic and optical properties. Examples of their mechanical capabilities are artificial muscles: in 2009 CNT actuators from aerogel sheets, drawn from forests of MWNTs, have been employed as a low-elastic modulus rubber when stretched in the sheet-width direction,[28] while in 2012 large-stroke, highpower, and high work-capacity yarn muscles that provide millions of cycles have been demonstrated, with a reversible actuation that is powered electrically, photonically or 


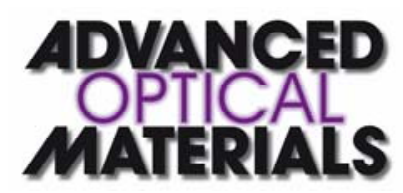

chemically (by adsorption and desorption).[29] In microelectronics, highly pure SWNT samples are attractive for transistors because of their low electron scattering cross section and their tunable bandgap, which depends on the diameter and the chiral angle. Moreover, SWNTs are well-suited for field-effect transistor designs and high-k dielectrics.[30] SWNT thin-film transistors are particularly attractive for driving organic light-emitting diode displays, because they have shown higher electrical mobility [31] than amorphous silicon and can be deposited by low-temperature, non-vacuum methods. Recently, flexible SWNT thinfilm transistors with a mobility of $35 \mathrm{~cm}^{2} / \mathrm{Vs}$ and an on/off ratio of $\approx 10^{6}$ were demonstrated.[31][32] SWNTs have large non-linear optical susceptibilities [33] and ultrafast carrier relaxation times [34][35] that make them attractive materials for ultrafast photonics, permitting all-optical switching in optical communication network devices and their employment as saturable absorbers in mode-locked lasers.[36][37] Promising applications in optoelectronics also include efficient photodetectors [38][39] and photovoltaic devices,[40] where CNTs can act both as active and passive materials. Photocurrent in CNT-based photodetectors can be either photovoltaic, typical of semiconducting CNTs, or thermal, in the case of metallic CNTs.[41] Recently, a CNT-graphene hybrid photodetector with photoresponsivity exceeding $100 \mathrm{AW}^{-1}$ and response time of $\approx 100 \mu$ s has been obtained.[39] On the other hand, photovoltaic devices made of a semiconducting CNT light-absorbing film and $\mathrm{C}_{60}$, acting as acceptor material, have reached internal quantum efficiencies of $\approx 80-$ $90 \% \cdot[42][43]$

This Review focuses on the elementary photo excitation dynamics in SWNTs as revealed by ultrafast spectroscopy: its aim is to summarize the current understanding of the overall photoexcitation scenario in SWNTs, including fundamental excitations, their deactivation pathways and the corresponding relaxation dynamics. This knowledge is an essential prerequisite in order to further promote the application of SWNTs in photonics and optoelectronics. In Section 2 we introduce the morphological and electronic properties of SWNTs and in Section 3 we list and discuss the different types of photogenerated species in SWNTs. After an overview of ultrafast spectroscopy techniques in Section 4, in Section 5 we report the ultrafast dynamics of the photoexcited species and coherent phonons in SWNTs, mainly focusing on the properties of semiconducting tubes. Finally, in Section 6 we present the open issues in the SWNTs photophysics and discuss how to address them with novel spectroscopic approaches. 


\section{Morphological and electronic properties of SWNTs}

Submitted to

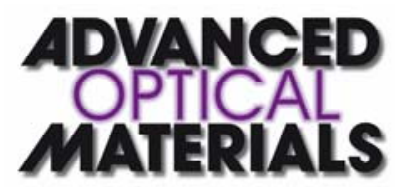

A SWNT has a honeycomb lattice of carbon atoms (graphene sheet) rolled into a hollow cylinder with nanometric diameter and with a length ranging between hundreds of nanometers and micrometers. The characteristics of SWNTs are intermediate between those of a single molecule and those of quasi-1D crystals with translational periodicity along the tube axis. As there are infinite ways of rolling the graphene sheet into a cylinder, defining the tube chirality, it is possible to produce a wide family of SWNTs with different microscopic structures, optical and electronic properties.[44] Since the morphology of SWNTs is intrinsically related to that of graphene, they are usually labeled in terms of the graphene lattice vectors. Their lattice constant is $a=\sqrt{3} a_{C C} \approx 2.46 \AA$, where $a_{C C}=1.42 \AA$ is the distance between carbon atoms in the honeycomb lattice. The basis vectors $\overrightarrow{\boldsymbol{a}}_{1}$ and $\overrightarrow{\boldsymbol{a}}_{2}$ depicted in Figure 1 are:

$$
\begin{aligned}
& \overrightarrow{\boldsymbol{a}}_{1}=\left(\frac{\sqrt{3}}{2} ; \frac{1}{2}\right) a \\
& \overrightarrow{\boldsymbol{a}}_{2}=\left(\frac{\sqrt{3}}{2} ;-\frac{1}{2}\right) a
\end{aligned}
$$

while the chiral vector $\overrightarrow{\boldsymbol{C}}_{h}$ is:

$$
\overrightarrow{\boldsymbol{C}}_{h}=n \overrightarrow{\boldsymbol{a}}_{1}+m \overrightarrow{\boldsymbol{a}}_{2} \equiv(n, m)
$$

with $0 \leq|m| \leq n$. From the chiral vector it is possible to calculate the tube diameter:

$$
d_{t}=\frac{\left|\overrightarrow{\boldsymbol{C}}_{h}\right|}{\pi}=\frac{a}{\pi} \sqrt{n^{2}+n m+m^{2}}
$$

that is approximately $0.85 \mathrm{~nm}$ for the $(6,5)$ chirality, depicted in Figure 1. 
(a)

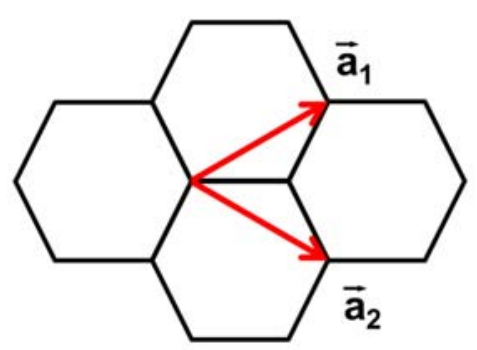

(b)

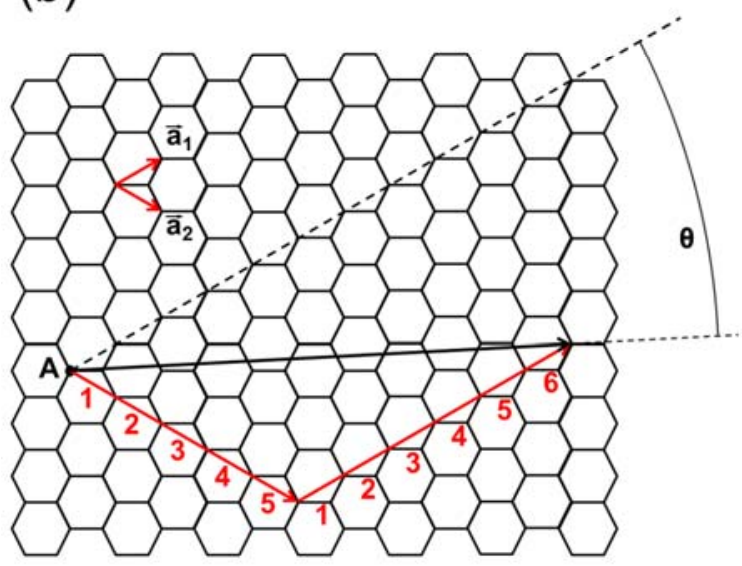

Figure 1. (a) Basis vectors $\overrightarrow{\boldsymbol{a}}_{1}$ and $\overrightarrow{\boldsymbol{a}}_{2}$ and (b) chiral vectors $\overrightarrow{\boldsymbol{C}}_{h}$ for $(6,5)$ SWNTs. $\theta$ indicates the angle between the zig-zag folding (dashed line) and the chiral vector for (6.5) SWNTs.

The easiest approach to the study of the electronic properties of SWNTs consists in applying circumferential boundary conditions to the band structure of graphene, according to the so-called zone-folding approximation. The electronic structure of graphene was first studied in 1946 by P. R. Wallace [45] and follows from a simple nearest-neighbour tight binding (TB) approximation, which already highlights its peculiar linear dispersion. Carbon has four electrons in the valence band; in graphene, three of them hybridize in the $2 \mathrm{sp}^{2}$ orbitals to create the carbon-carbon in-plane $\sigma$ bonds while the fourth $2 \mathrm{p}_{z}$ orbital creates the out-of-plane $\pi$ bonds, responsible for the graphene conductivity.[45][46] Thus graphene can be treated as a periodic structure having one single conduction electron in the $2 p_{z}$ state, which is the ideal condition (electrons located at isolated atomic sites) for the application of the TB approximation. The two atoms in the unit cell give rise to a valence $(\pi)$ band and a conduction $\left(\pi^{*}\right)$ band that touch at six points at the edges of the Brillouin zone. Due to symmetry, these points can be reduced to a pair of conical energy bands $\mathrm{K}$ and $\mathrm{K}$ ', independent of one another 


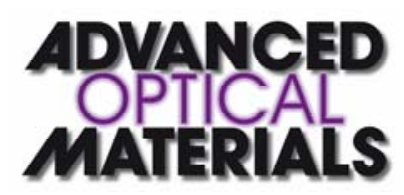

at the position in the reciprocal space. In fact, close to the $K$ and $K$ ' points the energy dispersion in graphene is linear:

$$
E(\boldsymbol{k})= \pm v_{F} \hbar \boldsymbol{k}
$$

where $v_{F}=\frac{3 t}{2 \hbar} a_{C C} \approx 10^{6} \mathrm{~m} / \mathrm{s}$ is the Fermi velocity and $t \approx 2.8 \mathrm{eV}$ is the nearest-neighbour hopping energy.[47] This dispersion relation corresponds to the Dirac equation

$$
E=c \hbar \boldsymbol{k}
$$

where the Fermi velocity $v_{F}$ replaces the speed of light $c$. Interestingly, the Dirac-like electronic spectrum of graphene is independent of the approximation considered and it is only a consequence of the symmetry of the lattice.[45] We will now exploit the graphene dispersion relation to understand the electronic properties of SWNTs; for a detailed description of the graphene electronic properties see ref. [47]. The most significant physical properties of SWNTs (such as electrical mobility, electrical and optical band-gap, phonon energy) strongly depend on their chirality and diameter, and this is a quite unique effect in solid-state physics. In fact, the boundary conditions along the circumferential direction of the SWNT give rise to a set of discrete values for the allowed wave vectors, while in the axial direction of the SWNT the wave vectors remain continuous (in the case of infinite tubes).[44] By plotting the allowed "circumferential" wave vectors in the Brillouin zone of a graphene sheet (Dirac cones at K and K' for valence and conduction bands), a series of parallel lines are generated, with length, number, and orientation that depend on the chiral indexes $(n, m)$ of the SWNT (Figure 2). This is the so-called zone-folding approximation, in which the electronic band structure of a SWNT with a specific chirality is given by the superposition of the graphene linear electronic energy bands along the corresponding allowed wave-vectors. For this reason, the electrical bandgap in SWNTs depends on the chiral angle: a nanotube is metallic if the allowed wave-vectors cross the zero-gap $K$ point in the graphene Brillouin lattice. This happens when $n-m=3 l$ (being $l$ an integer). Hence, 2/3 of the chiralities correspond to semiconducting SWNTs, while $1 / 3$ correspond to metallic (or semimetallic) ones.[44]

It is worth noting that for small diameter SWNTs the curvature is so strong that a rehybridization among $\sigma$ and $\pi$ states can occur, resulting in a significant alteration of their 
electronic structure with respect to the zone-folding approximation.[48] Coupling effects between adjacent tubes represent another possible source of alteration of the electronic properties. In fact, SWNTs are usually grown in bundles of tens to hundreds of tubes by several techniques such as arc-discharge, chemical vapour deposition or laser ablation.[44] Such bundles contain tubes with different chirality and diameter. Indeed, with advanced synthetic routes it is possible to obtain well separated single nanotubes, and therefore dispersions or films of almost identical SWNTs have been reported.[16][17][49][50] Coupling effects between tubes in bundles are evident, even in an ideal monodisperse bundle. In fact, it has been demonstrated by ab initio calculations that bundles of pure $(10,10)$ nanotubes (i.e. metallic tubes) already show the opening of a pseudo-gap of about $0.1 \mathrm{eV}$ at the Fermi energy.[51] Also ambient contamination and doping due to water and oxygen molecules, often a consequence of the SWNT production process, can strongly modify their electronic properties.[52][53]

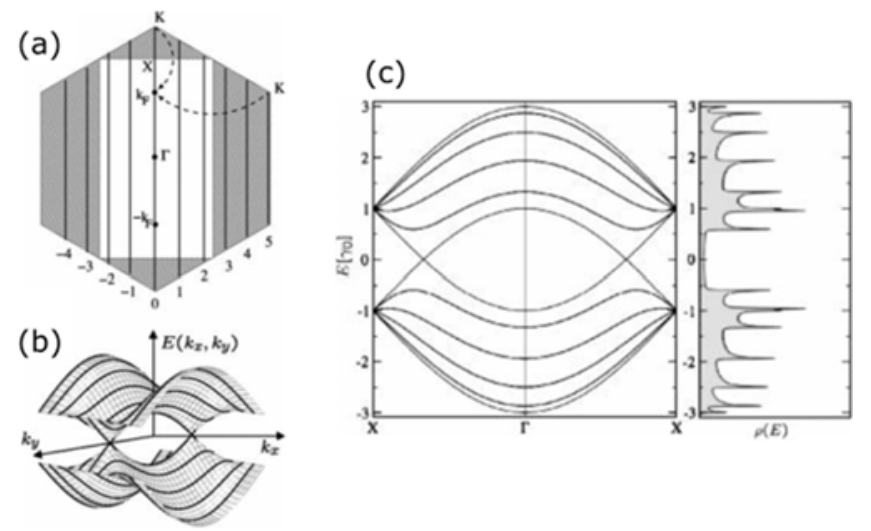

Figure 2. Electronic band structure for a $(5,5)$ metallic SWNT in the zone-folding approximation. (a) Brillouin zone of graphene with the allowed wave-vectors for the considered SWNT, (b) dispersion relation for the graphene plane and (c) resulting density of states. Reprinted with permission from [44].

\section{Photogenerated species in SWNTs}

The first optical studies on SWNTs were based on the assumption that their properties could be described by an independent free-electron model. In this picture, 1D quantum confinement leads to the creation of valence and conduction subbands and to the divergence of the density of states at the subband edges (Van Hove Singularities, VHS). Indeed, the intense peaks observed in the absorption spectra were related to the energy differences between VHS in the valence and conduction bands for a given tube chirality. The selection rule $\Delta q=0$, being $q$ the azimuthal quantum number, guarantees that the strongest optical 
Submitted to

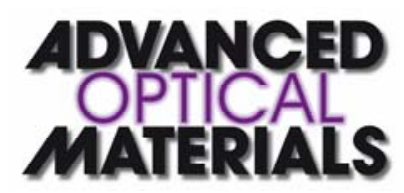

transitions take place between subbands with the same angular momentum.[54] Indeed, considering that the notation $E_{i j}$ (or $S_{i j}$ for semiconducting SWNTs) represents a transition from the $i$ valence subband to the $j$ conduction subband, the favored transitions are those with $i=j$, such as $E_{11}, E_{22}, E_{33}$ and so on. On the other hand, an optical excitation with polarization orthogonal with respect to the tube axis would lead to transitions between different subbands, such as $E_{12}$, however with much smaller absorption cross-section with respect to those for parallel polarization. Such studies were summarized in the well-known Kataura's plot [55] which collects on the same graph the $E_{i i}\left(d_{t}\right)$ transition energies as a function of the SWNT diameter $d_{t}$. The importance of this representation (Figure $\mathbf{3 b}$ ) stems from an early remark by White and Mintmire [56] that the electronic transitions should not depend on nanotube chirality but only on its diameter. The predicted trend results in well separated $E_{i i}\left(d_{t}\right)$ lines of the Kataura's plot.

(a)

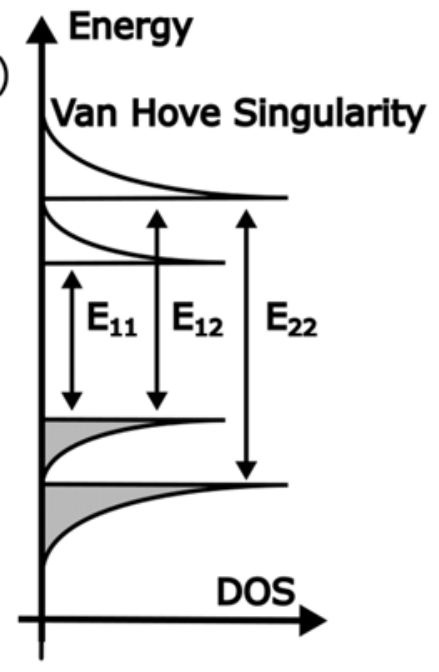

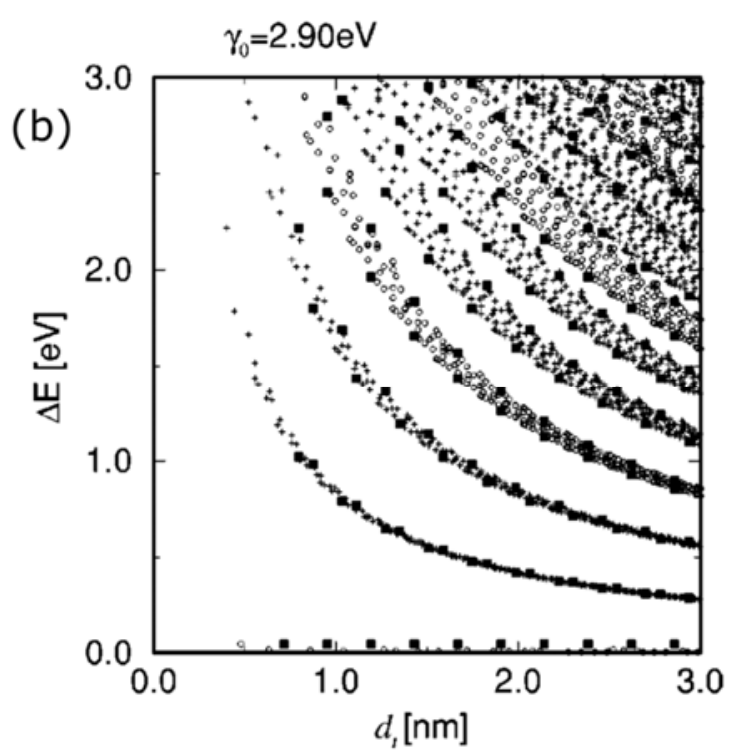

Figure 3. (a) Sketch of the $E_{i j}$ transitions, in the independent free-electron model, for a generic semiconducting SWNT. (b) Electronic transitions $E_{i i}(d)$ for all $(n, m)$ values as a function of the SWNT diameter $d_{t}$, with $0.7 \leq d_{t} \leq 3 \mathrm{~nm}$. Crosses and open circles denote the peaks of semiconducting and metallic tubes, respectively. Solid squares denote $E_{i i}(d)$ values for zigzag tubes. Here $\gamma_{0}$ is the nearest neighbor carbon-carbon interaction energy. Reprinted with permission from [57].

Although the diameter-only dependence of the transitions works remarkably well at low excitation energies, strong deviations can be observed in the Kataura's plot for high energy transitions, as experimentally confirmed by accurate linear absorption studies on enriched $(n, m)$ SWNTs.[58][59] In particular the ratio $E_{22} / E_{11}$, predicted to be equal to 2 in the approximation of linear bands close to the Fermi level,[60] was experimentally found to 
Submitted to

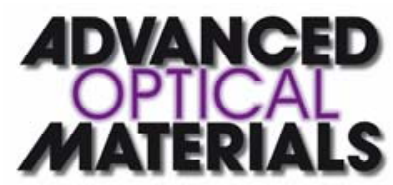

be significantly smaller,[58] leading to the so-called ratio problem.[61] Further, Bachilo et al.[58] reported the decrease of the photoluminescence (PL) intensity for SWNTs with small chiral angles (zigzag tubes), an effect that is hard to understand within the independentelectron model. These deviations motivated several theoretical studies investigating electron correlation and excitonic effects in SWNTs. In a simple 1D generalization of the MottWannier model [62] excitons show divergence of the binding energy of the electron-hole pairs [63] and quenching of the Sommerfeld factor which compensates for the VHS at the $E_{i i}$ edge. More accurate $a b$ initio calculations, confirmed by two-photon PL experiments [64][65] clearly showed that bound excitons, with binding energies of up to $1 \mathrm{eV}$, are primary photoexcitations in semiconducting SWNTs.[61][66][67][68] Such calculations were based on the solution of the Bethe-Salpeter equation in which also the electron-hole interactions are taken into account through the screened Coulomb interaction and an exchange term. This latter term also stabilizes triplet states, yielding potentially low-lying dark excitons with vanishingly small dipole matrix elements with the ground (singlet) state.[44]

Beyond excitons and triplets, also multiparticle species such as biexcitons and trions play a role in the photophysics of SWNTs, in particular in the limit of low inhomogeneous broadening and sufficiently high excitation fluence, such as those encountered in laser applications. Biexcitons are bound complexes formed by two excitons and are predicted to be surprisingly stable, having binding energies of approximately $50-100 \mathrm{meV}$ for small diameter SWNTs.[69][70] Trions, instead, are three-particle charged species arising from the combination of an exciton with either a hole or an electron. They are predicted to be stable and detectable at room temperature in optically excited junctions with injected carriers or in doped SWNTs.[71][72] Moreover, ultrafast photogeneration of free charges in semiconducting SWNTs upon excitation of the first excitonic transition has been experimentally demonstrated by means of transient absorption experiments, $[73][74][75][76]$ although in contrast with the excitonic model.

This discussion shows that the photoexcitation scenario in SWNTs is quite complex, with the possibility of several species being generated simultaneously, depending on the excitation conditions, the SWNT type and the timescale of observation. In Section 5 we will show how ultrafast optical spectroscopy can shed light into the nature and the fate of photoexcitations in SWNTs. 


\section{$4 \quad$ Ultrafast optical spectroscopy}

A number of ultrafast optical spectroscopy techniques, with time resolution from picoseconds down to a few femtoseconds, have been used to study the complex photophysics of SWNTs. Here, to facilitate the understanding of the results presented in Section 5 and to make this Review self-consistent, we provide a brief description of the most important experimental techniques that have been employed for SWNTs characterization.

\subsection{Pump-probe spectroscopy}

Pump-probe is the most versatile and widely used ultrafast spectroscopy technique and provides invaluable information on the nature and the dynamics of the photogenerated species in molecules and solids. The principle scheme of a femtosecond pump-probe experiment is shown in Figure 4. A first energetic pump pulse, resonant with an electronic (or vibrational) transition of the system under study, promotes some population from the ground to the excited state; the subsequent system evolution is monitored by measuring the transmission (or reflection) change of a delayed, weak probe pulse, as a function of the time delay $\tau$ between pump and probe pulses, typically controlled by a mechanical delay line. One usually detects the pump-induced variation of the probe energy measured by a slow detector, and the time resolution is determined by the instrument response function (IRF) of the apparatus, which is the cross-correlation between the intensity profiles of the pump and probe pulses.[77] The probe can be either an attenuated replica of the pump pulse, obtained by a beam splitter (degenerate pump-probe) or a pulse with a different colour (two-colour pumpprobe). In broadband (or hyperspectral) pump-probe spectroscopy [78] the probe is a broadband pulse, which is spectrally dispersed on an optical multichannel analyzer after the sample (Figure 4). One measures therefore a differential transmission $(\Delta \mathrm{T} / \mathrm{T})$ map, as a function of probe frequency $\omega_{\mathrm{pr}}$ and pump-probe delay $\tau$ :

$$
\frac{\Delta T}{T}\left(\omega_{p r}, \tau\right)=\frac{T_{o n}\left(\omega_{p r}, \tau\right)-T_{o f f}\left(\omega_{p r}\right)}{T_{o f f}\left(\omega_{p r}\right)}
$$

$\mathrm{T}_{\text {on }}$ and $\mathrm{T}_{\text {off }}$ being the transmission of the probe pulse with and without the pump pulse, respectively 


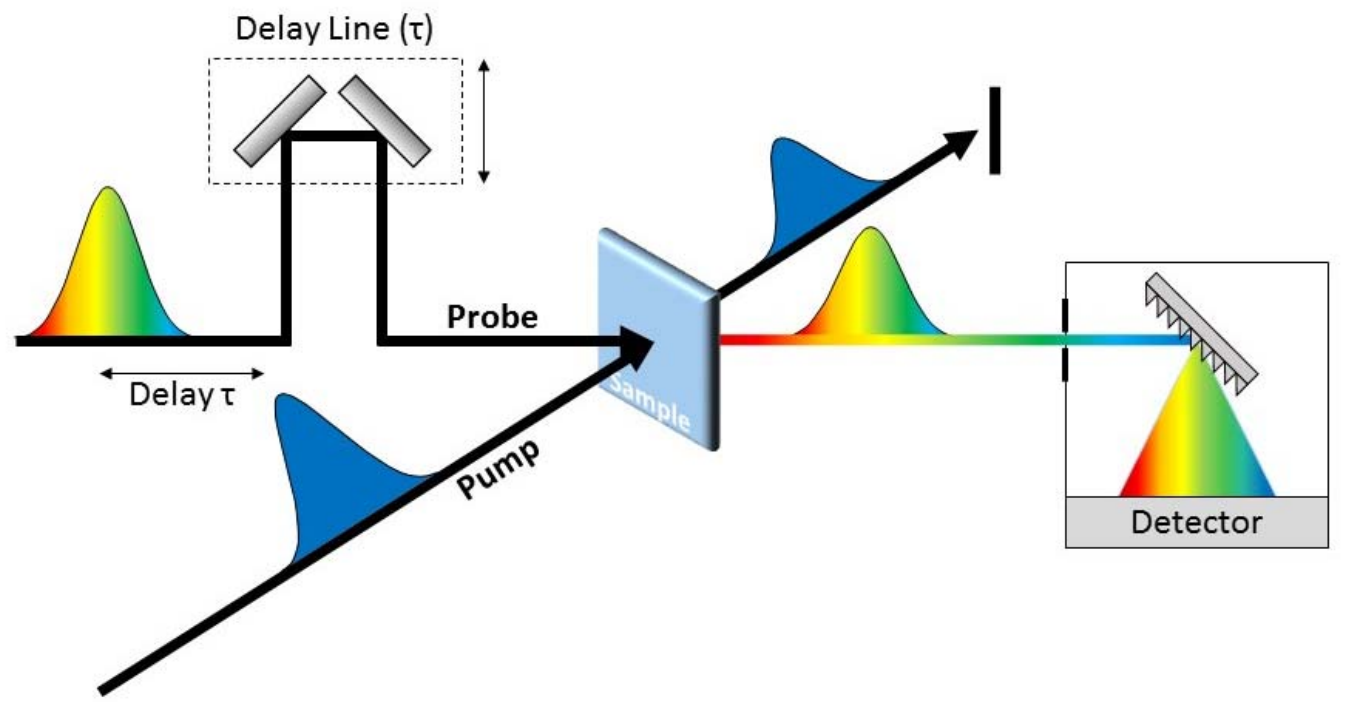

Figure 4. Scheme of a pump-probe experiment.

In order to relate the $\Delta \mathrm{T} / \mathrm{T}$ signal to the photoexcitation dynamics, let us write the sample transmission, according to Lambert-Beer's law, as:

$$
T(\omega)=e^{-\alpha(\omega) d}
$$

where $\alpha(\omega)$ is the frequency-dependent absorption coefficient and $d$ the sample thickness. For $\Delta \mathrm{T} / \mathrm{T}<<1$ we can easily derive:

$$
\frac{\Delta T}{T}\left(\omega_{p r}, \tau\right) \approx-\Delta \alpha\left(\omega_{p r}, \tau\right) d
$$

The absorption coefficient can be expressed as $\alpha(\omega)=\sum n_{i} \sigma_{i}(\omega)$, where $n_{i}$ are the populations (volume densities) of the ground state and the excited state(s) and $\sigma_{i}(\omega)$ are the corresponding absorption cross sections. We can then write:

$$
\Delta \alpha\left(\omega_{p r}, t\right)=\sum_{i}\left(\sigma_{i}\left(\omega_{p r}\right) \Delta n_{i}(t)+n_{i}(t) \Delta \sigma_{i}\left(\omega_{p r}, t\right)\right)
$$

The first term reflects the changes in the populations photoinduced by the pump pulse. The second term represents changes in the absorption cross section as a consequence of photoexcitation, which may be caused, for example, by the Stark effect due to photoexcited charges [79] or by bandgap renormalization.[80] Both terms will be important in the description of excited state dynamics of SWNTs. 
To understand the possible signals due to changes in populations, let us consider the system shown in Figure 5, which consists of a ground electronic state $(|0\rangle)$ and two excited states $(|1\rangle$ and $|n\rangle)$, each one with its vibronic progression. The pump pulse, resonant with the $|0\rangle \rightarrow|1\rangle$ transition, reduces the number of absorbers in the ground state, inducing, for a probe pulse with the same frequency, an absorption decrease; this is the so-called ground state bleaching (GSB), giving rise to a transmission increase $(\Delta T / T>0)$. At the same time, the pump pulse populates the excited state, so that a probe photon can stimulate it to emit back to the ground state; this stimulated emission (SE) signal, also causing a transmission increase $(\Delta \mathrm{T} / \mathrm{T}>0)$, occurs at probe frequencies equal or lower than the ground state absorption. For some probe frequencies GSB and SE overlap, while for others a pure SE signal is observed. Finally, the excited state populated by the pump pulse can absorb to the higher-lying level $|n\rangle$; this so-called excited state absorption (ESA) causes a transmission decrease $(\Delta \mathrm{T} / \mathrm{T}<0)$. ESA can occur at any probe frequency, depending on the energy level structure of the molecule under study; in particular, it can sometimes spectrally overlap the GSB and SE signals and even overwhelm them. Note that a negative $\Delta \mathrm{T} / \mathrm{T}$ signal, or photoinduced absorption (PA), is not always associated with an ESA; it can for example be due to absorption from a hot ground state [81] or to a change in the absorption cross section, such as a rigid shift, which leads to a transmission increase at some probe frequencies and decrease at others.

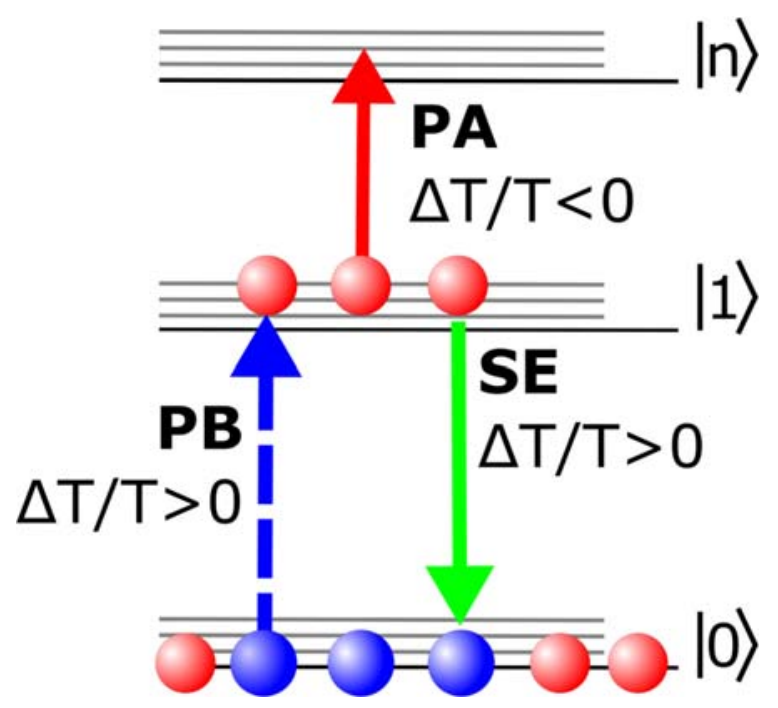

Figure 5. Energy levels scheme of a molecular system and possible signals in a pump-probe experiment. 
Submitted to

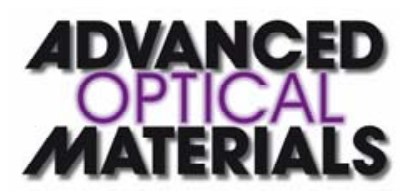

Experimental systems for pump-probe spectroscopy vastly differ in terms of sensitivity, temporal resolution and spectral coverage. Femtosecond oscillators, with their high repetition rates $\left(\approx 100 \mathrm{MHz}\right.$ ) allow high sensitivity (down to $\Delta \mathrm{T} / \mathrm{T} \approx 10^{-7}$ in combination with high frequency modulation of the pump) [82] but only limited spectral coverage, which can be extended by pumping optical parametric oscillators (OPOs). These high frequency systems are typically used in a degenerate configuration. Amplified system greatly increase the peak power at the expense of repetition rate (which decreases to $1-10 \mathrm{kHz}$ ) and allow the generation of broadly tunable pulses by driving one or multiple optical parametric amplifiers (OPAs). In particular, non-collinear OPAs (NOPAs) enable the generation of broadband, sub10-fs light pulses dramatically increasing the temporal resolution of the experiment.[83] Also amplified systems provide sufficient peak power for white-light continuum (WLC) generation in a transparent bulk material, such as a sapphire or a $\mathrm{CaF}_{2}$ plate; WLC, thanks to its superior stability and spatial beam quality, provides the ideal probe for hyperspectral pump-probe. Figure 6 shows the schematic of a typical pump-probe system with hyperspectral detection. It starts with an amplified Ti:sapphire laser, working at $1-10 \mathrm{kHz}$ repetition rate, whose output is split into two branches: one drives an OPA, generating the pump pulses, while the other generates a WLC probe by focusing into a sapphire (or $\mathrm{CaF}_{2}$ ) plate. Pump and probe are noncollinearly focused on the sample and the transmitted probe, spatially selected by an iris, is focused on a multichannel detector, typically an optical multichannel analyzer (OMA) capable of single-shot detection at the full laser repetition rate.[84] This system allows recording a $\frac{\Delta T}{T}\left(\omega_{p r}, \tau\right)$ map with $\approx 100$-fs temporal resolution and spectral coverage from the $\mathrm{UV}$ (400 nm with sapphire, $320 \mathrm{~nm}$ with $\left.\mathrm{CaF}_{2}\right)$ to the near-IR $(1.6 \mu \mathrm{m})$.

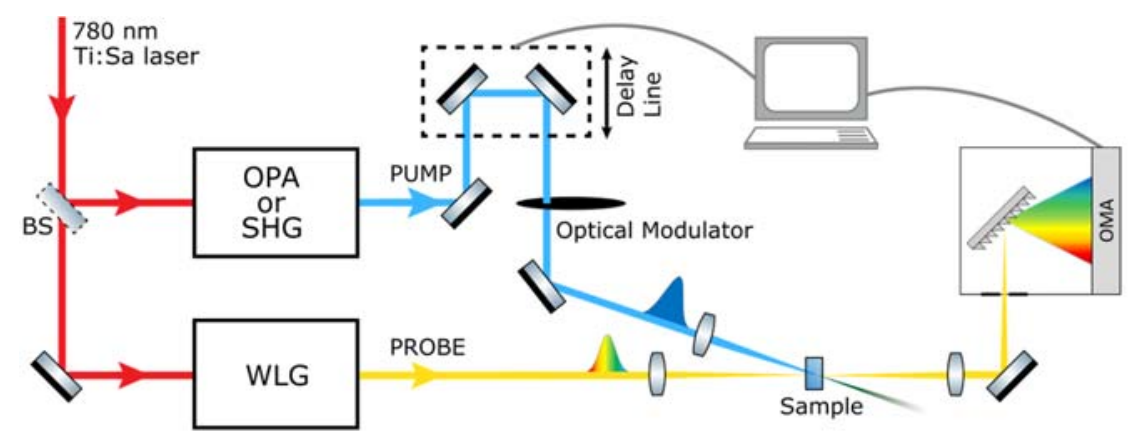

Figure 6. Femtosecond pump-probe setup with broadband detection using white light generation. BS, beam splitter; OMA: optical multichannel analyser; WLG: white light generation; SHG: second harmonic generation; OPA: optical parametric amplifier. 


\subsection{Photon-echoes and two-dimensional spectroscopy}

Pump-probe spectroscopy is a powerful tool for the study of population dynamics (or longitudinal relaxation time $\mathrm{T}_{1}$ ), but does not provide any information on the dephasing time of the photoexcitations (or transverse relaxation time $\mathrm{T}_{2}$ ). Dephasing can be measured by photon echo, which is a form of non-collinear degenerate four-wave-mixing experiment.[85][86] In a photon echo experiment the system interacts with a sequence of three non-collinear equal pulses, with relative delays $\tau$ and $T$ (see Figure 7): pulse 1 (with wave vector $\boldsymbol{k}_{\mathbf{1}}$ ) generates a polarization in the sample, which interferes with the field of pulse 2 (with wave vector $\boldsymbol{k}_{\mathbf{2}}$ ) creating, thanks to the non-collinear interaction geometry, a spatial population grating. Finally, pulse 3 (with wave vector $\boldsymbol{k}_{\mathbf{3}}$ ) interacts with the grating, giving rise to a third order nonlinear polarization $P^{(3)}(\tau, T, t)$ which radiates a field in the phase-matched direction $\boldsymbol{k}_{\mathbf{2}}-\boldsymbol{k}_{\mathbf{1}}+\boldsymbol{k}_{\mathbf{3}}$. The experiment measures the energy $\mathrm{U}_{\text {diff }}$ of the echo signal, which is

$$
U_{d i f f}(\tau, T)=\int_{-\infty}^{+\infty}\left|P^{(3)}(\tau, T, t)\right|^{2} d t
$$

Since the macroscopic polarization created by pulse 1 decays with the dephasing time $\mathrm{T}_{2}$, also the strength of the population grating will decay on a comparable timescale. Therefore, measuring the echo energy as a function of $\tau$ allows to obtain $T_{2}$, according to the expression:

$$
U_{\text {diff }}(\tau, T) \propto \exp \left(-\tau / 4 T_{2}\right)
$$

for the limit of large inhomogeneous broadening. If, on the other hand, one varies the delay of the third pulse $\mathrm{T}$, the echo energy measures the decay of the population grating:

$$
U_{\text {diff }}(\tau, T) \propto \exp \left(-T / 2 T_{1}\right)
$$

obtaining an information similar to pump-probe. If the three interactions occur with distinct pulses, we talk about three-pulse photon echo (3PPE);[86] if the second and third interaction occur with the same pulse (so that $\mathrm{T}=0$ and $\boldsymbol{k}_{\mathbf{3}}=\boldsymbol{k}_{\mathbf{2}}$ ) we talk about two-pulse photon echo (2PPE) [85] and the signal is emitted in the direction $2 \boldsymbol{k}_{\mathbf{2}}-\boldsymbol{k}_{\mathbf{1}}$. In some cases the time constant for the decay of the echo signal is comparable or shorter than the IRF of the apparatus, so that it becomes difficult to reliably extract it by deconvolution. In such case it is 
Submitted to

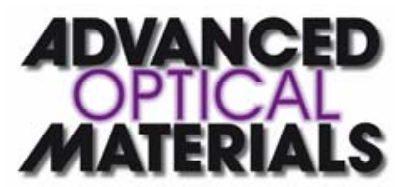

possible to measure simultaneously also the signal in the direction $\boldsymbol{k}_{\mathbf{1}}-\boldsymbol{k}_{\mathbf{2}}+\boldsymbol{k}_{\mathbf{3}}$ (or $2 \boldsymbol{k}_{\mathbf{1}}-$ $\boldsymbol{k}_{\mathbf{2}}$ for the 2PPE), which, since the roles of pulses 1 and 2 are reversed, is emitted for $\tau<0$. The signals in the two directions are mirror images with respect to $\tau=0$, and the time shift of their peaks (photon echo peak shift, PEPS) [87] can be shown to be proportional to $\mathrm{T}_{2}$. Measurements of the PEPS allow to reliably extract dephasing times even when they are much shorter than the IRF of the system.
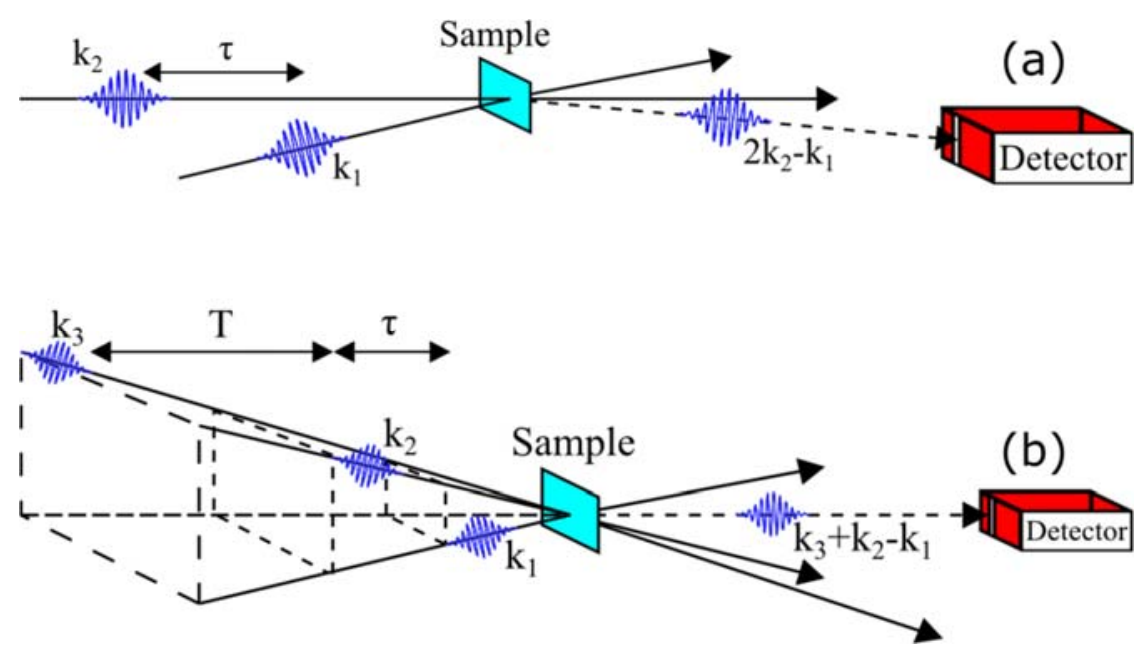

Figure 7. Two pulse (a) and three pulse (b) photon-echo schemes.

Two-dimensional electronic spectroscopy (2DES) [88] can be seen as a natural extension of photon echo spectroscopy, in which the echo signal is fully measured in amplitude and phase by optical heterodyning with an additional phase-locked pulse, the local oscillator (LO). This allows to fully retrieve the third-order polarization $P^{(3)}(\tau, T, t)$. By Fourier transforming with respect to $\tau$ and $t$ for a fixed value of the "waiting time" $T$, one obtains $\widetilde{P^{(3)}}\left(\omega_{\tau}, T, \omega_{t}\right)$ as a function of "excitation frequency" $\omega_{\tau}$ and "detection frequency" $\omega_{t}$. So far two schemes have been successfully used to implement 2DES in the visible range: the heterodyne detected 3PPE [89] and the partially collinear pump-probe geometry.[90] The heterodyne-detected 3PPE (Figure 8a) exploits the non-collinear interaction geometry between the three driving pulses to emit the four-wave-mixing echo signal in a backgroundfree direction, dictated by phase-matching. The echo signal, proportional to the third-order nonlinear polarization, is fully resolved in amplitude and phase using spectral interferometry with a fourth heterodyning pulse (the LO). The heterodyned signal is detected with a spectrometer, which optically Fourier transforms the signal generating the $\omega_{t}$ axis; a 
Submitted to

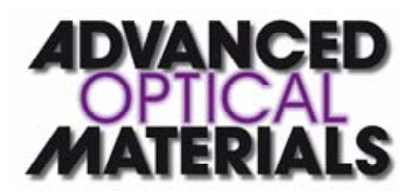

numerical Fourier transform with respect to $\tau$ is used to generate the $\omega_{\tau}$ axis. This scheme has the advantage of a background-free configuration but the drawback of requiring interferometric stabilization of two pulse pairs (pulses 1-2 and 3-4 respectively). In most implementation of 2DES, phase locking has been achieved by using as beam splitters diffractive optics,[89] or by active path-length stabilization.[91] The partially collinear pumpprobe geometry requires two phase-locked collinear pump pulses and a non-collinear probe pulse, which is dispersed on a spectrometer (Figure 8b). The probe pulse has the dual purpose of generating the nonlinear polarization and heterodyning it (self-heterodyning configuration). Advantages of this configuration are its simplicity and the fact that it automatically measures absorptive spectra, which are the most direct to interpret. An additional advantage of this geometry is that it easily lends itself to the extension to two-color 2DES. The collinear phase-locked pump-pulse pair can be generated by a balanced Michelson/Mach-Zehnder interferometer with active stabilization [92] or by a pulse shaper, which inherently provides interferometric stability due to the common path of the two pulses. Sinusoidal modulation of both amplitude and phase is required for the generation of a pulse pair. Several solutions, including liquid crystal spatial light modulators [93] acoustooptic modulators [90] and acousto-optic programmable dispersive filters [94] have been proposed. Finally, a passive interferometer using a sequence of birefringent wedges has been demonstrated.[95]
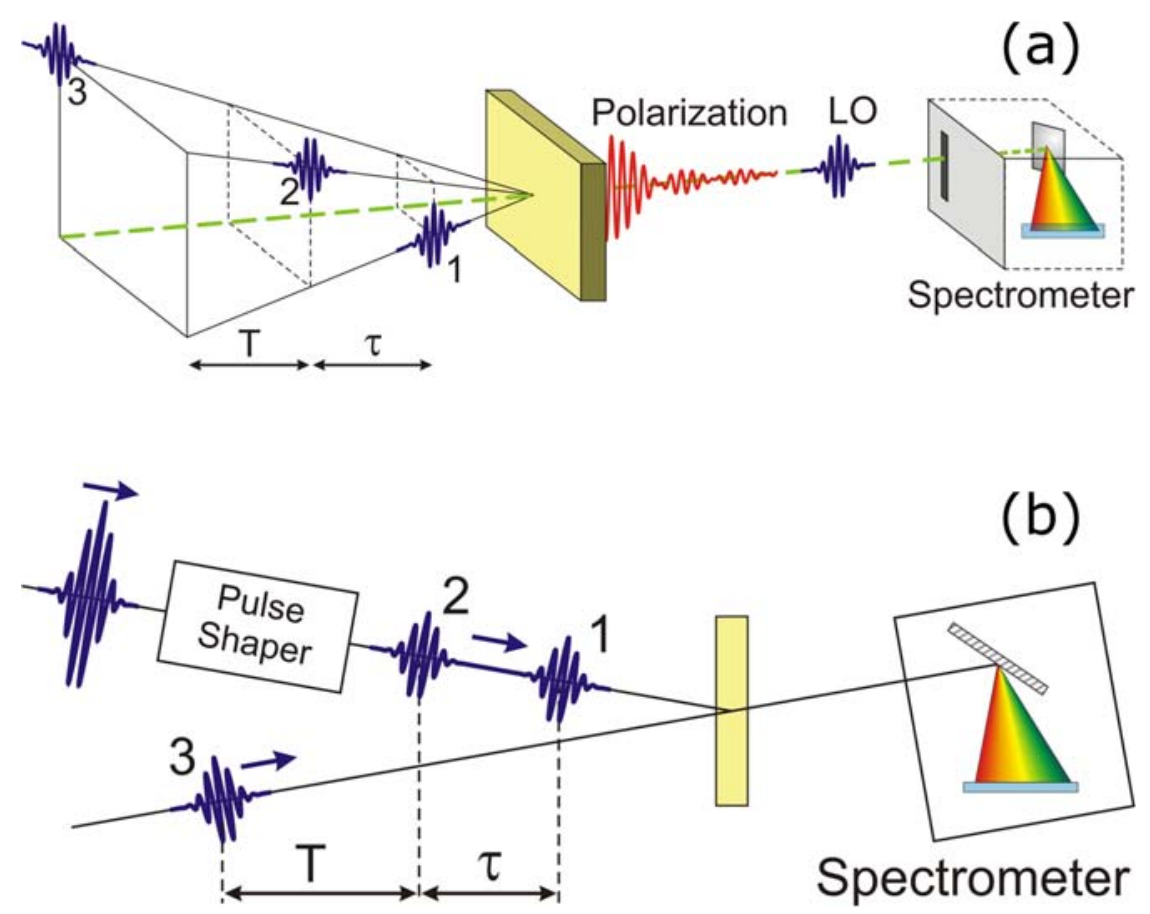
Submitted to

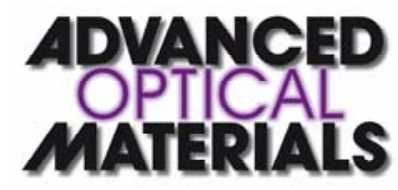

Figure 8. Scheme of the two experimental setups used for 2DES: (a) heterodyne detected three pulse photon echo and (b) partially collinear pump-probe geometry.

\subsection{Impulsive coherent vibrational spectroscopy}

Nowadays mode-locked Ti:Sapphire oscillators and NOPAs allow to produce light pulses with sub-10-fs duration, i.e. much shorter than typical periods of vibrations in molecules or phonons in solids. Photoexcitation with such short pulses generates coherent vibrational wavepackets in both the excited and the ground potential energy surfaces (PES).[96] Impulsive coherent vibrational spectroscopy (ICVS) enables direct time-domain detection of vibrational motions at frequencies as high as $2100 \mathrm{~cm}^{-1}$ (16 fs period).[97] To understand the generation of ground and excited state vibrational coherences, we recall that pump-probe spectroscopy, being a third-order nonlinear experiment, involves three field interactions with the sample.[98] The prominent term, bearing information on the material, has two field interactions with the pump, creating a population which is then interrogated by the probe field. For short pump pulses, excited state oscillations are observed when the two fields in the pump pulse excite a vibrational wavepacket (i.e. a coherent superposition of vibrational eigenstates) on the electronic excited state PES, which then subsequently oscillates back and forth leaving and returning to the Frank-Condon region. Ground state oscillations are generated when the first field induces a polarization wavepacket on the excited state PES, which is then allowed to propagate for some time so that the second field brings the wavepacket back down to the ground state, displaced from the hole left behind. This mechanism is known as Impulsive Stimulated Raman Scattering (ISRS).[99] In this case, the two sequential field interactions result in an impulsive resonant Raman process that transfers momentum from the light pulse to the ground state wave function using the excited state as an intermediary. In practice ICVS is performed in an hyperspecrtral pump-probe contifuration and measuring, for each probe frequency $\omega_{\mathrm{pr}}$, oscillations of the $\Delta \mathrm{T} / \mathrm{T}$ signal as a function of pump-probe delay $\tau$. By analyzing the probe wavelength dependence of the amplitude and phase of the coupled modes, ICVS provides insight on the structure of the ground and excited state PES.

\section{$5 \quad$ Ultrafast spectroscopy of SWNTs}

In this section we concentrate on the role of ultrafast spectroscopy in the study of the photophysics of SWNTs, with particular emphasis to the key experimental results that led 
Submitted to

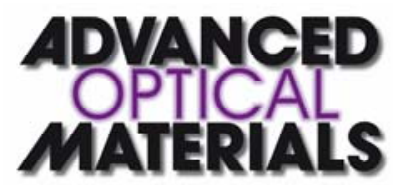

to the understanding of their intrinsic electronic and optical properties and to the more recent and still debated issues. In the first part we will discuss the experiments which have allowed to establish the excitonic nature of the SWNTs optical transitions and to reveal their intrinsic properties (e.g. exciton binding energy, size, mobility) and dynamics. Subsequently we will study other photo-excited species (e.g. charges, triplets, biexcitons) with particular attention to their role in the optical response of the sample and finally we will present time-domain studies of the SWNTs vibrational response.

\subsection{Excitons}

\subsubsection{Binding energy}

The most convincing experimental evidence regarding the excitonic nature of SWNTs' optical resonances and their binding energy was obtained by two-photon excited PL (2PPL).[64][65][100] The presence of sharp peaks in the linear absorption spectrum of SWNTs, in fact, can be attributed either to excitons or to the presence of VHS in a 1D confined semiconductor, and a simple linear experiment can hardly distinguish between these two interpretations. On the other hand, a two-photon absorption experiment can distinguish between states of different symmetry in the Rydberg series of an excited state. In fact, in analogy to the 3D hydrogen atom, the excited states, in the case of $1 \mathrm{D}$ excitons, can have odd $(u)$ or even $(g)$ symmetry, which correspond to the $s$ and $p$ orbitals of the hydrogen atom model. Due to optical selection rules, a one-photon transition accesses the $1 s$ state while a two-photon absorption ends up on the $p$-symmetry excited state (Figure 9). A 2PPL experiment gives simultaneous information about the two-photon absorption energy (i.e. the energy of the accessed even $2 p$ excited state) and the corresponding one-photon PL emission energy (i.e. the single-photon emission and absorption of the $1 s$ state). By combining this information with the energy difference between the $2 p$ state and the continuum (also obtained by the 2PPL experiment) one can derive the exciton binding energy (i,e, the energy difference between the continuum and the 1s state). Experiments were performed exciting the SWNTs with tunable ultrashort pulses in the near infrared (0.6-1 eV photon energy) generated by either an OPA [64] or an OPO [65] and detecting the corresponding 2PPL. The evidence of a large energy gap, of the order of hundreds of $\mathrm{meV}$, between the two-photon absorption and the PL emission energies was decisive to rule out the band picture of optical transitions in SWNTs and confirm their excitonic nature. This experiment also allowed to obtain the 
exciton binding energy for a large number of semiconducting SWNTs,[101] which turns out to be several hundreds of $\mathrm{meV}$, i.e. a significant fraction of the SWNT bandgap. This result should be compared to typical binding energies for the excitons in 3D semiconductors, which are of the order of a few meV,[102] i.e. much smaller than $\mathrm{kT}$ at room temperature. Therefore excitons are the dominant photoexcitations in SWNTs even at room temperature, while they are completely ionized in bulk seminconductors. Moreover, Wang et al. [64] observed a broad energy linewidth of the $2 p$ state that they attribute mainly to the ultrafast decay from the $2 p$ to the $1 s$ state. An ultrafast pump-probe experiment with two-photon excitation, being able to follow in real time the relaxation of the $2 p$ state, could thus provide new insights into the fundamental properties of excitons. The excitonic nature of SWNTs optical transitions was demonstrated also by means of femtosecond pump-probe experiments,[103][104][105] with the observation of an extremely rapid exciton-exciton annihilation [105] and the appearance of ESA bands originating from transitions between the first exciton $\mathrm{S}_{11}$ and the onset of the continuum [104] or between excitonic excited states.[103]

While the two lowest lying excitons $\left(\mathrm{S}_{11}\right.$ and $\left.\mathrm{S}_{22}\right)$ in semiconducting SWNTs are strongly bound, Raman experiments have highlighted significant differences for the higher lying excitonic transitions,[106][107][108] suggesting that they might arise from free electron-hole pairs. The nature of these transitions is still a matter of debate, since a simple tight binding model completely fails in the assignment of the $S_{33}$ transition energy [57] and predicts binding energies [67] even larger with respect to $S_{11}$. Experiments, on the contrary, suggest very low or even vanishing binding energies, although it is still not clear whether the $\mathrm{S}_{33}$ is excitonic in nature.[107][109] Recently, we obtained a binding energy of $\approx 80 \mathrm{meV}$ for the $\mathrm{S}_{33}$ exciton in $(6,5)$ semiconducting SWNTs [110] by means of broadband pump-probe spectroscopy. 


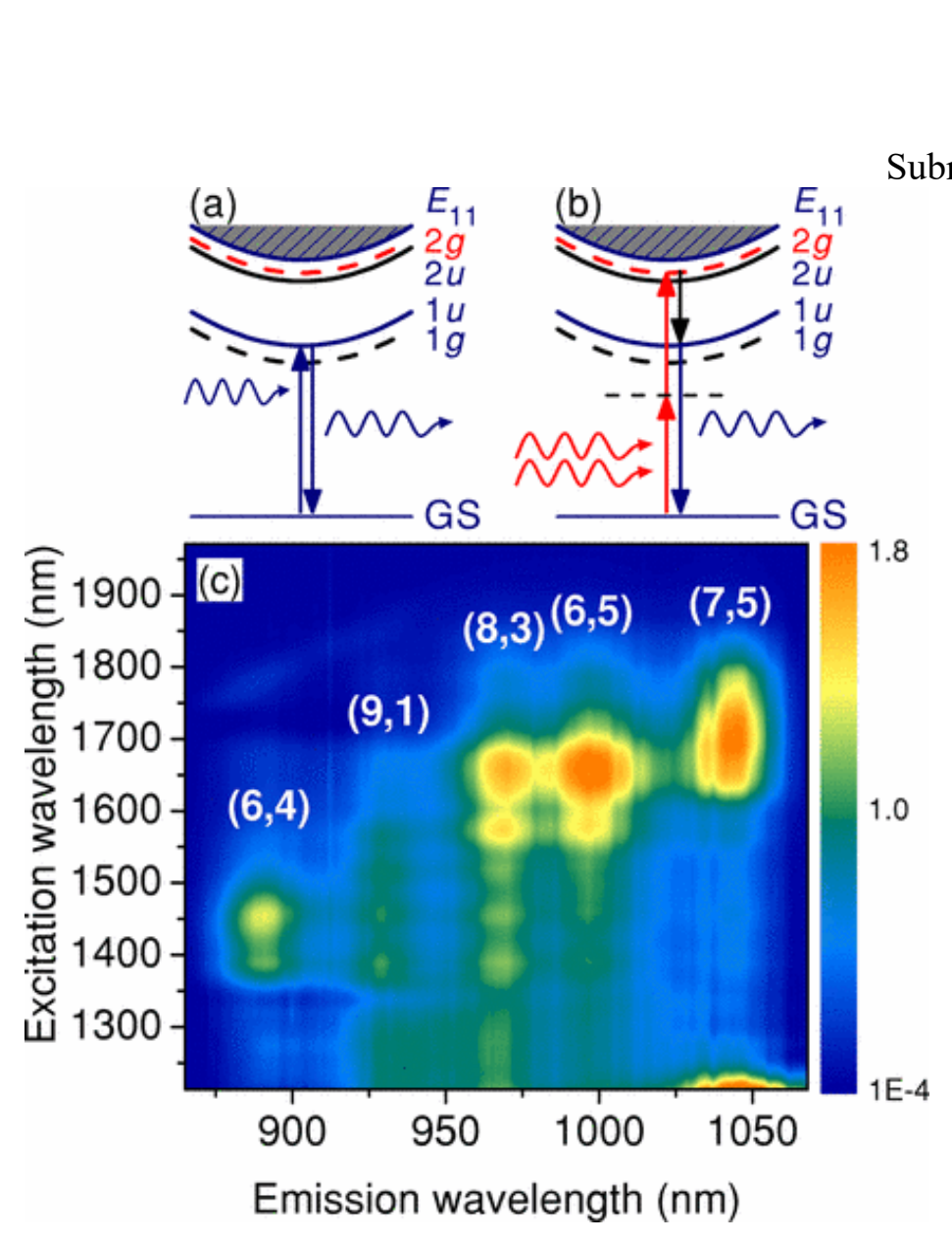

Figure 9. Schematic picture of a one- and two-photon PL experiment. $E_{11}$ indicates the single-particle transition between the lowest subbands, namely the first VHS obtained with tight binding calculations. a) A one-photon absorption ends up in an excited state with odd symmetry $(1 u$ or $1 s)$ while b) twophoton absorption creates an excited state with even symmetry $(2 g$ or $2 p)$. Emission always occurs from the lowest one-photon active state. c) Two-photon luminescence spectra of carbon nanotubes of different chiralities, plotted as a function of excitation and detection (emission) wavelength. Reprinted with permission from [65].

\subsubsection{Size and mobility}

Having established by 2PPL that the primary photoexcitations in SWNTs are excitons, ultrafast pump-probe experiments were crucial to determine the exciton size and mobility. The electron-hole distance (or correlation length, or "exciton size") gives direct information about the electronic structure and physical properties of the material, such as screening, Coulomb attraction, binding energy, exchange interaction and confinement of wave-functions. Moreover, measurement of the exciton size allows distinguishing between the loosely bound Wannier-Mott excitons, typical of covalent semiconductors where the electron-hole distance is much larger than the lattice constant, and the tightly bound Frenkel excitons, typical of molecular solids, where the electron-hole distance is comparable to the lattice constant. Theoretical works [68][111] predicted an exciton size in the order of 1-2 nm, which was experimentally confirmed in the work of Wang et al. [64] by an approximate 
Submitted to

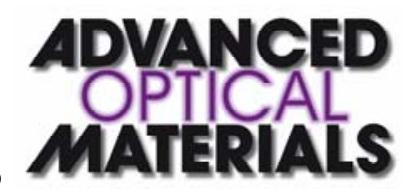

estimation of $R=1.2 \mathrm{~nm}$, being $R$ the electron-hole distance, thus confirming the picture of a strongly bound exciton. A more precise and detailed estimation of the exciton size, in $(6,5)$ SWNTs hails from the work by Lüer et al..[112] Using pump-probe spectroscopy with $\approx 15$-fs temporal resolution, it was possible to measure the GSB of the $\mathrm{S}_{11}$ transition before the onset of any relaxation dynamics. The exciton size was then obtained by a pump fluence dependent measurement (Figure 10) of this GSB signal interpreted in view of the phase space filling theory.[113] This experiment confirmed an exciton size of $2 \pm 0.7 \mathrm{~nm}$, in agreement with theoretical predictions; this value, which should be compared to the lattice constant of 0.14 $\mathrm{nm}$, definitely proved the conjecture that excitons in SWNTs can be described by a WannierMott picture. Based on the same intensity dependent measurements and considering excitonexciton annihilation in the exciton rate equations, Lüer et al. [112] were able to measure also an exciton diffusion coefficient of $D \sim 0.1 \mathrm{~cm}^{2} \mathrm{~s}^{-1}$. This value is much smaller with respect to other values obtained from pump-probe depolarization spectroscopy,[103] fluorescence quenching,[114] CW and time-resolved fluorescence spectroscopy.[115] This discrepancy can be explained in terms of the temporal resolution of the different experiments, which probe species with different mobility: an ultrafast pump-probe experiment is able to probe the initial population, i.e. the short-lived excitons that are also the most abundant, while photoluminescence is sensitive to long-lived excitons, which show much higher diffusion coefficients
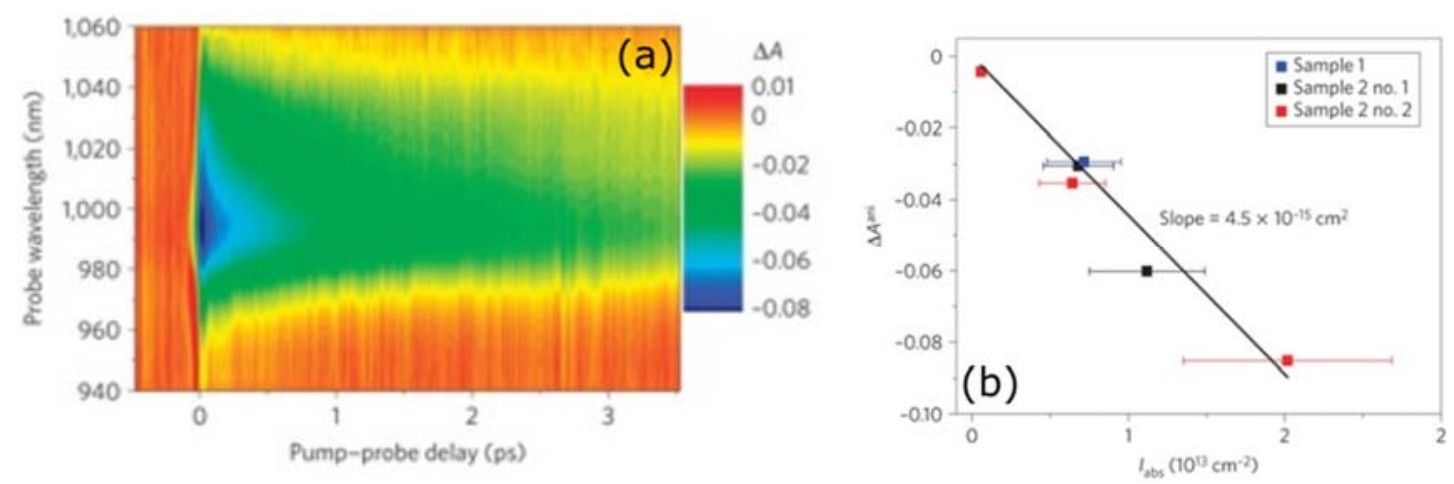

Figure 10. a) Two-dimensional $-\Delta \mathrm{T} / \mathrm{T}$ spectrum in the $\mathrm{S}_{11} \mathrm{GSB}$ of a $(6,5)$ enriched SWNT sampleafter pumping with $15 \mathrm{fs}$ pulses centered at 960 . b) Maximum differential absorption $\Delta \mathrm{A}$ from the pumpprobe spectra at zero pump-probe delay as a function of the absorbed pump photon fluence in the linear regime. The solid line is a linear regression through the origin. The experiment was done with different samples, as indicated. The error bars reflect the uncertainty in the incident light fluence. Adapted from Reference [112].

\subsubsection{Dephasing time}


Submitted to

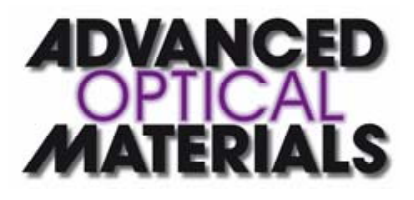

Information on the exciton dephasing times in SWNTs was obtained by photon echo and 2DES experiments. The earliest experiments of 2PPE [116] and 3PPEPS [117] on semiconducting SWNTs demonstrated that the first excitonic subband $S_{11}$ is inhomogeneously broadened. The homogeneous linewidth of the transition derives from both fluence dependent exciton-exciton interactions and temperature-dependent exciton-phonon interactions. A more recent and detailed analysis by Graham et al. [118] confirms these results and examines in depth all the contributions to the dephasing time. The dephasing time $T_{2}$, i.e. the time on which the polarization of the exciton transition persists after photoexcitation, is inversely proportional to the homogeneous linewidth, that is related to the pure dephasing time $T_{2}{ }^{*}$ and to the population decay time $T_{1}$ through the relation $1 / T_{2}=$ $1 / 2 T_{1}+1 / T_{2}{ }^{*}$. Typically, $T_{1}$ is much longer with respect to $T_{2}{ }^{*}$ and thus $T_{2} \sim T_{2}{ }^{*}$. This is not true for SWNTs, where the fastest decay component of the first exciton, which typically causes the relaxation of more than $50 \%$ of the total excited population, is of the order of the dephasing time. Accordingly photon-echo experiments, which probe only the dephasing time $T_{2}$, are not enough for the investigation of all the contributions to the homogeneous line-width and pump-probe measurements are required in order to extrapolate the population decay time $T_{1}$. At room temperature Graham et al. [118] obtained $T_{2}=205 \mathrm{fs}, T_{1}=372 \mathrm{fs}$ and $T_{2}{ }^{*}=$ $283 \mathrm{fs}$. Although this relatively high value of $T_{2}{ }^{*}$, which is typical of a low exciton-phonon coupling, might be unexpected in SWNTs (due to their well-defined vibrational modes and large surface area exposure to the surrounding environment), it is in good agreement with the high motional narrowing retrieved from the temperature dependent $2 \mathrm{PPE}$ decay profile. The dephasing time of the second excitonic subband $S_{22}$ for a $(6,5)$ SWNT was instead retrieved with a one-color pump-probe experiment.[119] Fitting with a third-order polarization model the saturation behavior of the pump-probe signal, Schneck et al. obtain a total dephasing time $T_{2}=36 \mathrm{fs}$. This is dominated by the pure dephasing time $T_{2}{ }^{*}$ while the ultrafast component of the population time $T_{1}$ remains unvaried over the explored pump fluences. Graham et al. [120] performed 2DES on the $\mathrm{S}_{22}$ transition of the $(6,5)$ semiconducting SWNTs using 12-fs pulses from a NOPA. The 2DES maps display cross peaks that correspond to the energy transfer from the phonon sidebands to the $\mathrm{S}_{22}$ state, mediated by second-order exciton phonon coupling processes. These measurements demonstrate that such phonon sideband states are indeed bound to the singlet $\mathrm{S}_{22}$ exciton and help to shed light into non-radiative decay processes in SWNTs. 


\section{a \\ Submitted to \\ MATERIALS}

\subsubsection{Exciton dynamics}

The understanding of exciton dynamics and their relaxation pathways is of fundamental importance in view of possible applications of SWNTs in photonics and optoelectronics. Ultrafast optical spectroscopy is extremely useful in the determination of all the processes that are involved in the radiative and non-radiative relaxation, taking into consideration interactions with phonons, defects and impurities, charges or other excitons. Despite the large body of available experimental work, still the scenario is not fully understood, due to the complexity of the system itself, with its set of direct or indirect photoexcited species such as charges, biexcitons, trions, triplets,[121] and the strong effect of different environments.[122] Some key experimental results help clarifying the situation; in the following we will try to derive a schematic representation of SWNTs excited state dynamics based on inter- and intra-band transitions and exciton-exciton interactions.

Early ultrafast spectroscopy studies on semiconducting SWNTs [103][123][124] established that, when excited to higher energy levels, they rapidly relax to the lowest energy exciton, on a timescale comparable to the typically available $100-\mathrm{fs}$ temporal resolution. This inter-subband $\left(\mathrm{S}_{22} \rightarrow \mathrm{S}_{11}\right.$ and $\left.\mathrm{S}_{33} \rightarrow \mathrm{S}_{11}\right)$ relaxation dynamics was first resolved by two-color pump-probe spectroscopy with few-optical-cycle pulses,[35] namely by direct excitation of the second and third excitonic transition $\left(\mathrm{S}_{22}\right.$ and $\left.\mathrm{S}_{33}\right)$ and detection of the first exciton $\left(\mathrm{S}_{11}\right)$ buildup

Figure 11). In this experiment short-pulse NOPAs were used for both excitation and detection. The relaxation dynamics are retrieved both from the delayed formation of a high energy PA signal (interpreted as an ESA from $\mathrm{S}_{11}$ to higher lying excitonic subbands [103]), as shown for the dynamics at $2.15 \mathrm{eV}$ in

Figure 11d, and the delayed buildup of the $\mathrm{S}_{11}$ exciton GSB, as shown for the dynamics at $0.92 \mathrm{eV}$ in

Figure 11c, upon excitation of $S_{22}$ in the visible spectral range. On the contrary, these two signals form instantaneously upon excitation of $\mathrm{S}_{11}$ at $0.92 \mathrm{eV}$ (

Figure 11a and b). This experiment defines the time scale $(\approx 40 \mathrm{fs})$ of the ultrafast intersubband relaxation, with longer recovery times for higher excitation energy (i.e. larger excess energy to be dissipated). Non-resonant excitation at higher energies with respect to the first excitonic subband also relaxes into the lowest available excitonic energy site,[125] but very little is known about the internal conversion mechanisms.[40] Recently, near-infrared PL 
Submitted to

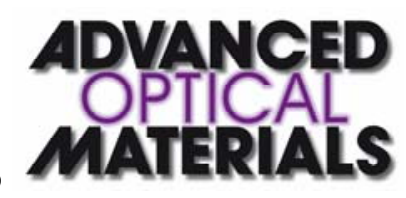

upon photoexcitation at an energy well below that of the exciton emission at room temperature was also demonstrated.[126] This was attributed to efficient one-phonon-assisted up-conversion processes enhanced by accidentally or intentionally embedded localized states.
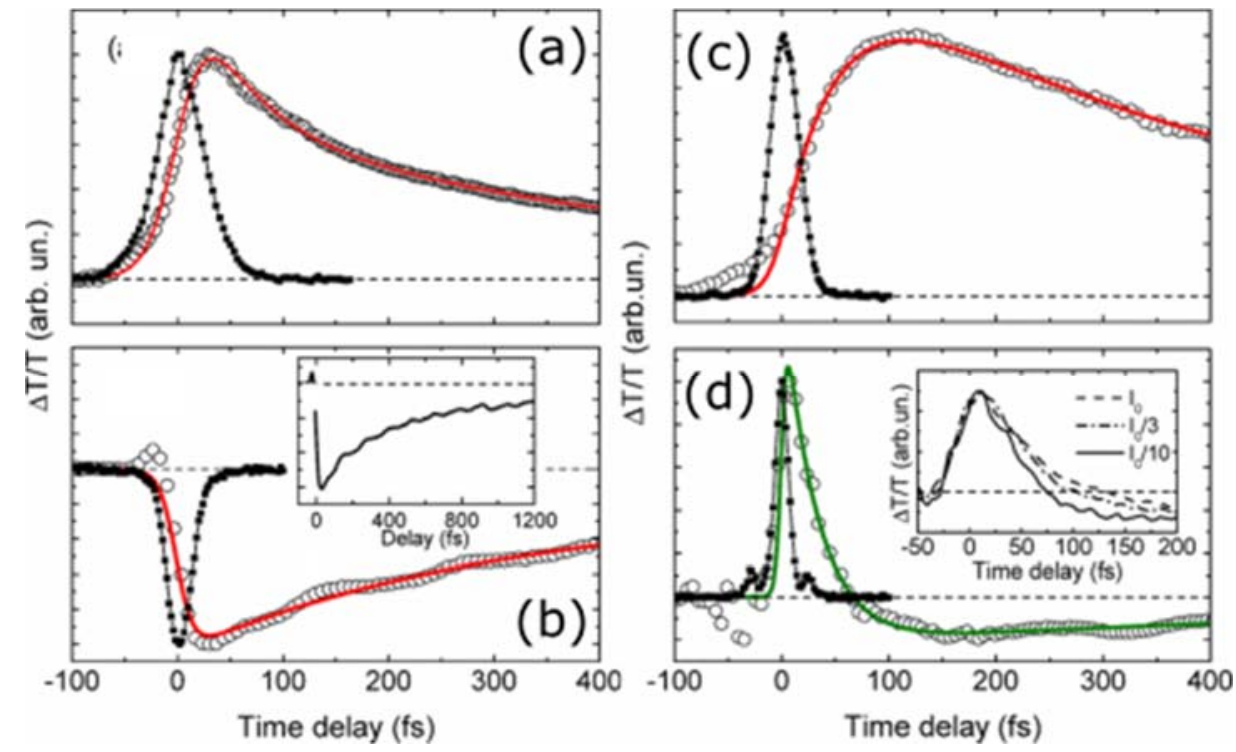

Figure 11. $\Delta \mathrm{T} / \mathrm{T}$ dynamics of SWNTs excited at $0.92 \mathrm{eV}$ and probed (circles) at a) $0.95 \mathrm{eV}$ and b) 2 $\mathrm{eV}$. The figure also shows (solid line) the fit and the pump pulse autocorrelation (squares). The inset in (b) shows the measurement on a longer time scale. To the right, differential transmission dynamics of SWNTs excited in the visible and probed (circles) at c) $0.92 \mathrm{eV}$ and d) $2.15 \mathrm{eV}$. Inset in (d) shows intensity dependence of the dynamics at $2.15 \mathrm{eV}$. Adapted from Reference [35].

After the ultrafast internal conversion to $\mathrm{S}_{11}$, the temporal evolution of semiconducting SWNTs is governed by the dynamics of the lowest excitonic transition. A striking properties of SWNTs is their very low PL quantum yield, of the order of $\eta=10^{-4} \div 10^{-}$ ${ }^{3}[59][127][128]$ which indicates the presence of efficient non-radiative decay channels. From theoretical estimates of the radiative decay time in SWNTs $\tau_{\text {rad }} \approx 100 \mathrm{~ns}$, based on the oscillator strength of the excitonic transitions,[127] one therefore gets for the non-radiative decay time $\tau_{\mathrm{nr}} \approx 10 \div 100 \mathrm{ps}$, in good agreement with experimental observations. In the following we will therefore concentrate on decay processes up to $\approx 100 \mathrm{ps}$. According to the excitation fluence, the excitonic population dynamics can be described by a two- or threecomponent exponential decay.[129][130] Chou et al. [130] found, for a sample of $(6,5)$ enriched SWNTs, three time constants $\tau_{\text {fast }} \sim 700 \mathrm{fs}, \tau_{\text {int }} \sim 2-3 \mathrm{ps}$ and $\tau_{\text {slow }} \sim 50 \mathrm{ps}$ (Figure 12). The fast sub-ps dynamics strongly depends on the pump fluence and it is usually dominated by bimolecular Auger processes.[105][131][132] The Auger process (also known as exciton-exciton annihilation) is a two-exciton interaction process, in which one exciton 
Submitted to

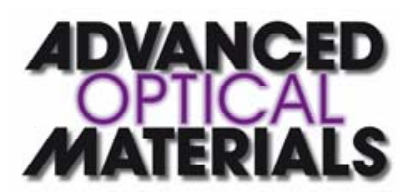

recombines to the ground state and the other is promoted to a higher excitonic state or dissociates into a free electron-hole pair (see Figure 13).

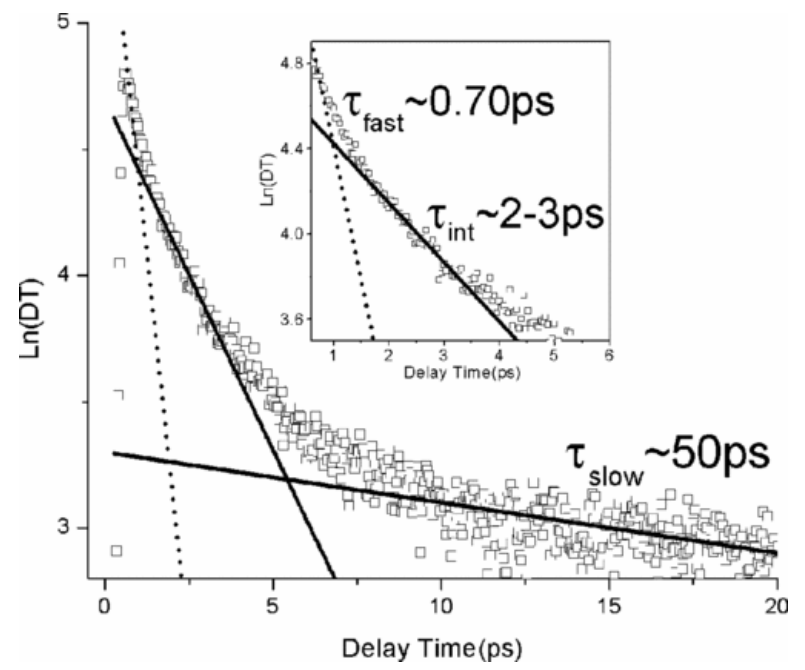

Figure 12. Differential transmission (in natural $\log$ scale) for the first exciton $S_{11}$ in a $(6,5)$ SWNT. The three decay components $\left(\tau_{\text {fast }}, \tau_{\text {int }}, \tau_{\text {slow }}\right)$ are due to Auger recombination, D-phonon mediated decay and exciton radiative lifetime respectively. Reprinted with permission from [130].

For a 1D system such as SWNTs the annihilation process can be described by the following rate equation for the exciton concentration nexc:

$$
\frac{d n_{e x c}}{d t}=-\gamma t^{-1 / 2} n_{e x c}^{2}
$$

where the $\mathrm{t}^{-1 / 2}$ dependence of the annihilation rate reflects the variable distance between the interacting excitons.[133] In fact the maximum annihilation rate corresponds to the distance between nearest neighbors, while the rate decreases as the excitons diffuse away from each other with time. The opposite process of bimolecular exciton-exciton annihilation is Multiple Exciton Generation (MEG), which was demonstrated in SWNTs by Wang et al..[134] MEG, also known as carrier multiplication, consists in the generation of two excitons following absorption of one photon with energy higher than twice the bandgap (see Figure 13). MEG was identified in $(6,5)$ SWNTs by following the appearance of a sub-ps dynamics at very low pump fluences when the excitation photon energy is higher than twice the energy gap; a quantum yield for MEG of 110(130) \% was measured for excitation at 400(335) nm. The MEG process, enhanced by the strong electron-hole confinement in SWNTs, is particularly promising for photovoltaic applications, as it would allow to obtain higher photon-to-current 
conversion efficiencies with respect to the Shockley-Queisser limit.[135] Recent experiments on ultra-narrow graphene nanoribbons (GNRs) found an extremely efficient exciton-exciton annihilation rate,[136] in good agreement with the values obtained for SWNTs.[105][131][132] This result clarifies the fundamental importance of the reduced dimensionality for this nonlinear relaxation mechanism.
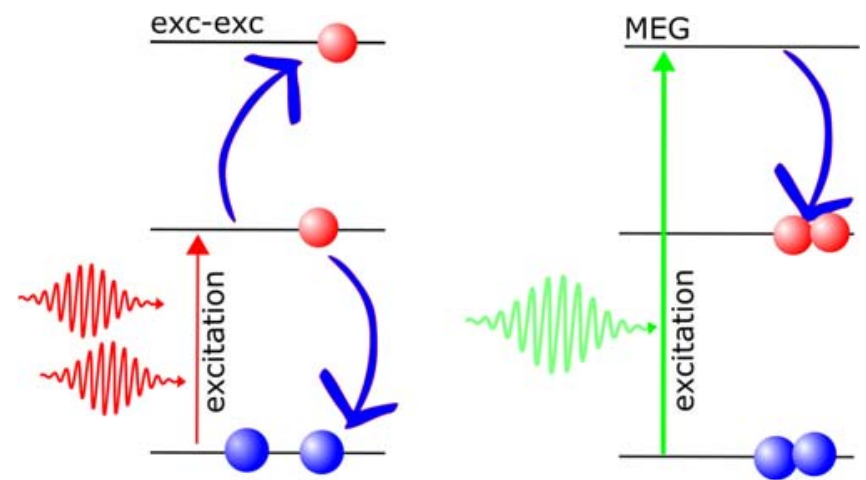

Figure 13. Sketch of exciton-exciton annihilation (left) and MEG (right) processes. In the high excitation regime due to exciton-exciton annihilation one exciton recombines to the ground state while another is promoted to a higher energy level. Multiple Exciton Generation is the dual of this process, in which, upon absorption of a high energetic photon, two low-energy excitons are formed.

The intermediate, picosecond decay component was attributed to a phonon-mediated process, in which the absorption of a D-phonon determines the trapping of the exciton into a dark state.[130][137] Finally, the slow decay component contains contributions from both a weak radiative recombination process and a dominant phonon-assisted relaxation process [130] and it appears to be stronger in samples with strong PL. [138]

A more direct measurement of SWNTs exciton lifetime can be obtained with time-resolved PL. In fact, pump-probe experiments are sensitive to both the emitting and the non-emitting species, so that the generation of long-lived non-emitting species such as triplet excitons or charge carriers would still result in the GSB of the excitonic transition; timeresolved PL experiments, on the other hand, only measure the excited states which emit light. Experimental results on isolated SWNTs obtained mono- [127][139] and bi-exponential [140] decays with PL lifetimes ranging from tens to hundreds of $\mathrm{ps}$ and highlighted the importance of non-radiative decay channels associated to the presence of trap states.[139] Such trap states can be either extrinsic, i.e. due to tube defects or intrinsic, i.e. due to the fine structure of the excitonic transition. It has in fact been predicted theoretically that the band edge exciton is split into two states, one bright (of odd parity) and one dark (of even parity), located a few 


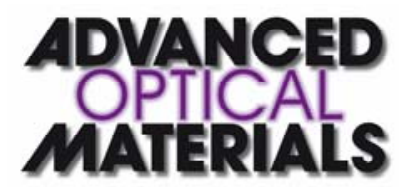

meV below the bright one; the two states thermalize via interaction with acoustic phonons.[140] The existence of a broad and featureless low-energy (below the optical bandgap) absorption band, that we have recently measured experimentally,[141] might further explain the importance of the non-radiative recombination channels.

Interestingly, in some cases ultrafast pump-probe experiments have also observed power law, i.e. $\Delta T / T \propto t^{-0.5}$, instead of multi-exponential decay dynamics for the excited state recombination in semiconducting SWNTs. Such dynamics is characteristic for geminate recombination following a random walk in a 1D system [142] and has been attributed to either triplet-triplet annihilation,[143][144] diffusion limited exciton-exciton recombination [145] or one-dimensional geminate recombination of photogenerated free charges.[110]

The picture presented so far focuses only on the excitonic signature in ultrafast pumpprobe experiments. The ultrafast dynamics of SWNTs, however, cannot be satisfactorily described by just considering the excitonic signal. In fact, the exciton GSB signal, commonly used for the study of exciton dynamics, is actually a consequence of ground-state depletion or filling of the free-carrier continuum of states and thus it does not descend directly from exciton generation. This leads to the risk of confusing the exciton lifetime with the time needed for ground-state recovery of other photo-excited species, such as free e-h pairs, which still bleach the excitonic transition. In the next section we will discuss the role of the different photo-excited species by following their spectroscopic signature in pump-probe experiments.

\subsection{Beyond excitons}

In the two decades since their discovery, the interpretation of the optical and electronic properties of SWNTs has dramatically evolved. As previously discussed in section 3 , the first studies of this ideal 1D system used a tight-binding model and led to the idea that VHS and free carriers were responsible for both the optical and the electronic response. The evolution of this early conjecture opened to the study of new photo-excited species, from excitons $[64][65][66]$ to biexcitons,[70][146][147] triplets [148][149] and trions.[71][72][150] In addition, evidence of the generation of free charges upon photoexcitation has also been obtained.[73][74][75] This complex scenario is still far from being fully understood and complicates the interpretation of ultrafast pump-probe experiments.

A bi-exciton (XX) is a four-particle state consisting of two bound excitons and has been predicted to have a large binding energy (of the order of $100 \mathrm{meV}$ ) in SWNTs.[151] Colombier et al. [70] detected the presence of biexcitons in SWNTs embedded in a gelatine 
Submitted to

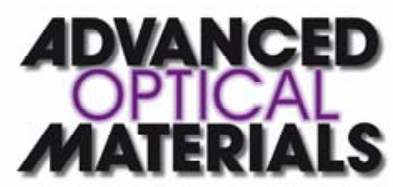

matrix by means of nonlinear optical spectroscopy, reporting a binding energy for the $(9,7)$ tube of $106 \mathrm{meV}$, in excellent agreement with theoretical predictions. The spectroscopic signature of the bi-exciton is an ESA band, corresponding to the $\mathrm{X} \rightarrow \mathrm{XX}$ transition, which is red-shifted with respect to the $\mathrm{S}_{11}$ transition by an amount corresponding to the bi-exciton binding energy. The exciton to bi-exciton transition was also used to interpret the ESA bands observed in pump-probe experiments on individual chirality selected SWNTs.[147][152] Recently, bi-excitons with comparable binding energies have also been identified in GNRs,[136] which share many of the spectroscopic properties of SWNTs. Charged excitons, or trions $\left(\mathrm{X}^{*}\right)$, are another class of many-body excitations and consist to an exciton which is bound to a charge, either an electron (negative trion) or an hole (positive trion). Similarly to bi-excitons, 1D quantum confinement in SWNTs leads to an increase in the trion binding energy.[72] Trions in SWNTs were detected by measuring the PL spectra of individual tubes at high excitation densities.[150] In addition to the emission from the singlet exciton and a phonon sideband, a red-shifted chirality dependent peak is observed, which is attributed to emission from trions $\left(\mathrm{X}^{*}\right)$, in which the charge is formed by bimolecular exciton-exciton annihilation. The trion binding energy, depending on the tube chirality, ranges between 100 and $200 \mathrm{meV}$. Park et al. [148] studied polymer-wrapped (6,5) SWNTs and demonstrated the formation of triplet states by rapid intersystem crossing (ISC), with time constant $\tau_{\text {ISC }} \approx 20$ ps and moderate efficiency $\left(\eta_{\mathrm{ISC}}=5 \pm 1 \%\right)$. These triplet states are long-lived $\tau_{\mathrm{T}} \approx 30 \pm 10 \mu \mathrm{s}$ and their spectroscopic signature is a PA band red-shifted with respect to the $\mathrm{S}_{11}$ band and corresponding to the $\mathrm{T}_{1} \rightarrow \mathrm{T}_{\mathrm{n}}$ transition. Stich et al. [143] found triplet-triplet annihilation to be responsible for delayed fluorescence from SWNTs. Both $\Delta \mathrm{T} / \mathrm{T}$ and time-resolved PL signals display a $\mathrm{t}^{-1 / 2}$ power-law decay which is characteristic of diffusion-limited annihilation in a $1 \mathrm{D}$ system. The above discussed complex photoexcitation scenario in SWNTs is summarized in Figure 14. 

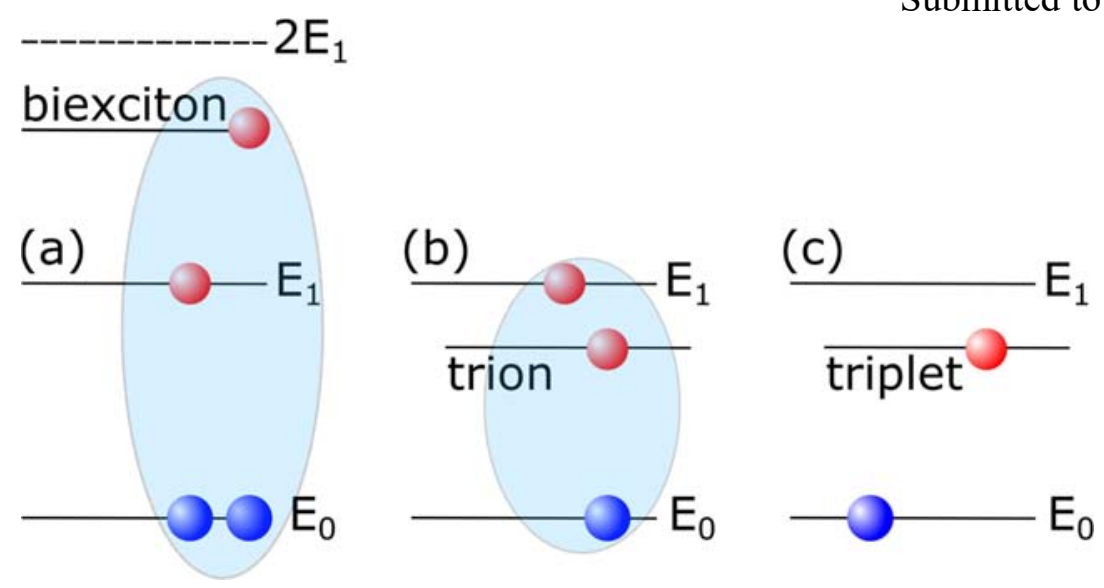

Figure 14. Sketch of three different possible photoexcited species in carbon nanotubes. (a) Biexciton: neutral quasi-particle formed by two strongly bound excitons; (b) trion (or charged exciton): charged quasi-particle formed by one electron (or hole) and on exciton; (c) triplet: unboud electron-hole pair with a spin of 1 .

Evidence for the photogeneration of free charges in SWNTs was obtained from broadband pump-probe spectroscopy. The different excitons, in fact, show completely different behaviors in their $\Delta \mathrm{T} / \mathrm{T}$ response, ranging from a sharp GSB of the first excitonic $\left(\mathrm{S}_{11}\right)$ transition to a complex shape for higher lying excitons $\left(\mathrm{S}_{22}\right.$ and $\left.\mathrm{S}_{33}\right)$. In particular, it is clear that a negative $\Delta \mathrm{T} / \mathrm{T}$ signal is not always related to an ESA [103] and instead it can be interpreted in terms of broadening or energy shift of the ground state absorption spectrum, corresponding to the $\Delta \sigma_{i}$ term in Eq. (9. This experimental observation has been reported in a number of studies, although with very different interpretations.[74][146][147][153][154][155] This suggests that different energy regions can be used as a direct probe of the different photo-excited species, and hyperspectral pump-probe appears particularly useful to this purpose thanks to its simultaneous high temporal resolution and broad spectral coverage.
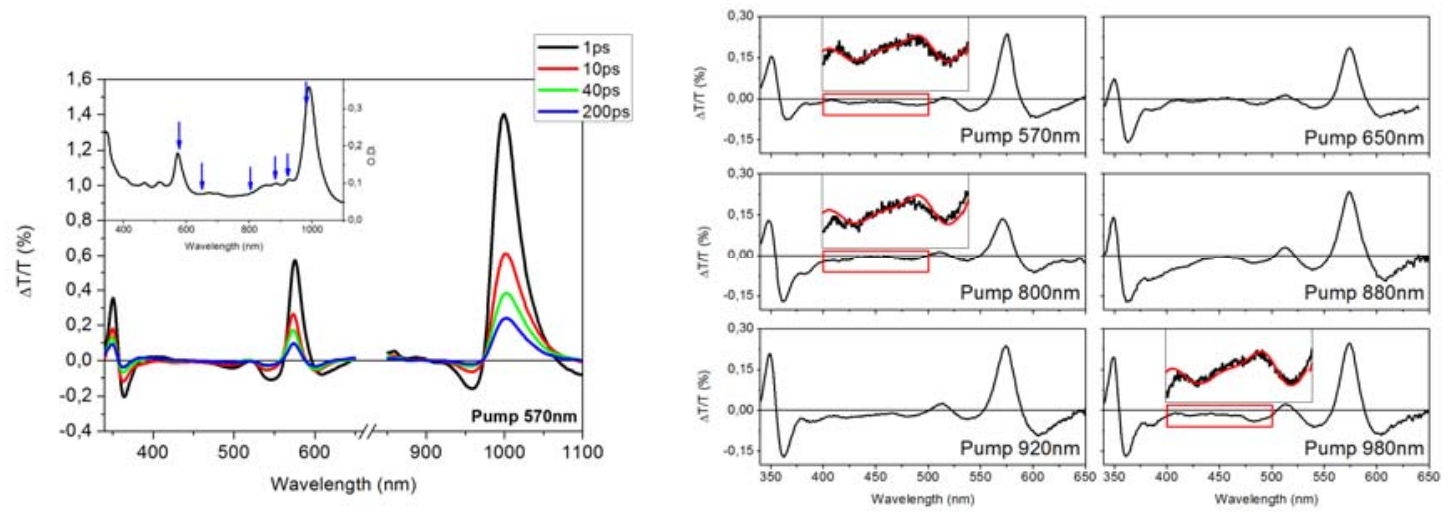

Figure 15. (left) $\Delta \mathrm{T} / \mathrm{T}$ spectra at different pump-probe delays with $570 \mathrm{~nm}$ excitation wavelength for an enriched $(6,5)$ semiconducting SWNT (inset for the ground state absorption spectrum). (right) $\Delta \mathrm{T} / \mathrm{T}$ 


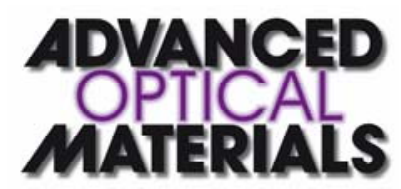

spectra at 30 ps pump-probe delay for six different excitation wavelengths, resonant and non-resonant with excitonic transitions.

For the following discussion we use the semiconducting $(6,5)$ SWNTs as an example. Figure 15, depicting the $\Delta \mathrm{T} / \mathrm{T}$ signal at different delays for an extremely broad probe wavelength range from $340 \mathrm{~nm}$ to $1.1 \mu \mathrm{m}$, shows a clear difference between the transient optical response of the first three excitons. The signal on $\mathrm{S}_{11}$ is dominated by a sharp GSB, regardless of the excitation (resonant or non-resonant) photon energy. The origin of this GSB is trivial when the excitation wavelength is resonant with the first excitonic subband (degenerate pump-probe) and can be easily interpreted as a phase space filling due to ultrafast relaxation to the lowest energy level or as a depletion of a common ground state when the pump energy is resonant with higher lying excitons (two-color pump-probe). When the excitation energy is above the first excitonic peak but far from any excitonic resonance of the most abundant chirality, this GSB was explained in terms of energy migration within SWNT bundles.[125] Both to the red and to the blue of the $\mathrm{S}_{11}$ GSB there are PA signals, which are more evident in pure samples where they do not overlap with signals arising from different chiralities. The blue-shifted PA signal for a pure $(6,5)$ SWNT is extremely long-lived (up to ms timescale) and it was assigned to charge induced diameter deformation.[156] At lower energy with respect to $\mathrm{S}_{11} \mathrm{PA}$ signals can have different origins. The lowest energetic ESA is the one related to absorption from the $S_{11}$ to the edge of the continuum of states [104][125] which also corresponds to the exciton binding energy of the nanotube under investigation. According to the purity of the sample, then, one could find short-lived transitions due to absorption of Dirac fermions in metallic tubes,[125] extremely long-lived ESA from triplet states [148] or PA due to trions formation.[121][150] More recently experiments on individual metallic SWNTs [152] detected blue and red shifted PA that were attributed to biexciton formation. These PA signals are surprisingly more intense with respect to those observed in semiconducting SWNTs near $\mathrm{S}_{11}$ although the exciton binding energy for metallic tubes is typically smaller.[157]

The $\Delta \mathrm{T} / \mathrm{T}$ response of the second and higher lying excitons in semiconducting SWNTs is even more complex. Near $\mathrm{S}_{22}$ two sharp and intense PA peaks appear to the red and blue side, and with comparable amplitude, with respect to the exciton GSB. This peculiar shape has been interpreted in terms of broadening due to scattering between carriers in different subbands,[153] bi-exciton formation,[146][147] phonon relaxation [155] or Stark effect.[74][154] Interestingly, the $\Delta \mathrm{T} / \mathrm{T}$ signal displays a shape that is perfectly reproduced by 
Submitted to

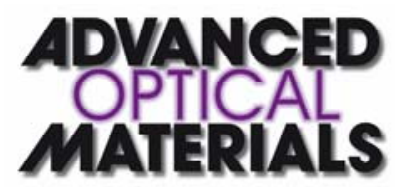

a red-shift of the ground-state absorption spectrum regardless of the excitation energy and in a very large probe region to the blue of the second exciton (Figure 15). The red-shift of the entire absorption spectrum, and not only of the excitonic resonances, obtained both for resonant and non-resonant excitation, suggest charge-induced Stark effect as a more likely interpretation for the observed signal.[74][154][158]

The shape of the $\Delta \mathrm{T} / \mathrm{T}$ response for the third exciton (Figure 15), near $350 \mathrm{~nm}$, is surprisingly similar to the first derivative of the ground state absorption spectrum and extremely long-lived, thus confirming the observed trend of decreasing GSB and increasing PA weight for increasing probe photon energies. This high energetic probe region appears to be ideal for tracking photogenerated free-carriers, being almost insensitive to $\mathrm{S}_{11}$ excitons and strongly shifted by charge-induced Stark effect due to the low binding energy of

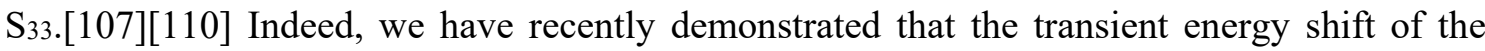
highly polarizable $\mathrm{S}_{33}$ transition is an extremely sensitive fingerprint of charge-carriers in SWNTs and its time decay is well described by a $t^{-1 / 2}$ power law, in agreement with geminate charge recombination in a $1 \mathrm{D}$ solid.[110]

The study of charge photogeneration in SWNTs is crucial for photonics and optoelectronics applications, since unintentional carrier photogeneration is detrimental for applications that require high exciton photogeneration yield and long lifetimes, such photovoltaics devices. Direct excitation of free carriers is accessible with high energy photons [154] while possible mechanisms for dissociation of the lowest excitonic transitions are still largely discussed. Recent experiments [159] show that $\mathrm{S}_{22}$ is more likely to undergo dissociation into free electron-hole pairs with respect to relaxation into $\mathrm{S}_{11}$. Nevertheless, $\mathrm{S}_{22}$ lies in the continuum of states of $\mathrm{S}_{11}$ and thus exciton dissociation processes are more likely, while dissociation of $\mathrm{S}_{11}$ was predicted to occur only in the presence of intense external electric fields.[160] Experiments on charge photogeneration upon excitation of $\mathrm{S}_{11}$ are indeed controversial: photo-current (PC) measurements show both field-induced exciton dissociation [161][162] and direct detection of free carriers without any external driving force;[74][163] $\mathrm{THz}$ experiments predict linear exciton dissociation or instantaneous free carriers generation;[73][75] pump-probe experiments at high excitation fluences show charge photogeneration after exciton-exciton annihilation.[150] Interestingly, recent PC experiments on isolated semiconducting SWNTs show the presence of free carriers upon excitation of $\mathrm{S}_{11}$ but they exclude field-induced exciton dissociation as a mechanism of charge photogeneration.[164] Moreover, ambient contamination, such as oxygen or water molecules, 
Submitted to

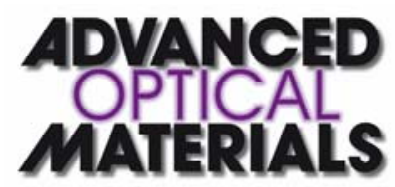

can act as dopant for SWNTs and lead to dramatic changes in their optical [100] and electronic properties.[52][53] By means of flash-photolysis time-resolved micro-wave conductivity, Park et al. [76] have recently found that charge photogeneration spontaneously occurs in individually suspended $(7,5)$-chirality-enriched SWCNTs for both $\mathrm{S}_{11}$ and $\mathrm{S}_{22}$ excitation. This results was obtained at low excitation fluences $(\approx 0.05$ excitons per $\mu \mathrm{m}$ length of tubes) and for SWNTs dispersed in a low dielectric solvent, thus suggesting that direct excitation more than exciton dissociation is responsible for charge photogeneration in SWNTs. Both these mechanisms, i.e. exciton dissociation and direct charge photogeneration, can be explained by the presence of a below-gap continuum of states, that we recently observed in semiconducting $(6,5)$ SWNTs.[141]

Free carriers might also play a role in energy transfer processes in SWNTs bundles,[165][166][167] which are ideal candidates for photovoltaic devices. Recently, Mehlenbacher et al. [168] have addressed energy transfer in SWNTs by performing 2DES experiments on films of blended SWNTs which consist of the $(7,5),(7,6),(8,6)$ and $(8,7)$ chiralities. In fact, due to its capability to correlate different transitions, 2DES can be used to probe energy transfer pathways in networks of SWNTs. Using WLC both for excitation and as a probe, they could address the $S_{11}$ transitions of all chiralities, which lie in the near-IR between 1000 and $1350 \mathrm{~nm}$. Figure 16 reports a sequence of 2DES maps for different values of the waiting time T. At $\mathrm{T}=0.1 \mathrm{ps}$ the signals on the diagonal correspond to the response of the individual chiralities, with the GSB of the $\mathrm{S}_{11}$ transition and the associated PA. The diagonal peaks are clearly elongated, indicating large inhomogeneous broadening of the transitions. At later delays, cross peaks appear which correspond to downhill energy transfer to SWNTs of smaller bandgap, with all the energy in the end arriving to the $(8,7)$ chirality. The cross peaks are round, indicating uncorrelated energy transfer between different chiralities. Exponential fitting of the rise of the cross peaks yields time constants between 1 and $3 \mathrm{ps}$. The fact that the transfer rate appears to be unrelated to the spectral overlap between donor emission and acceptor absorption allows to rule out Förster mechanisms and to assign the transfer process to exciton tunneling, possibly mediated by diffusion to "hot spots" where two tubes cross. 


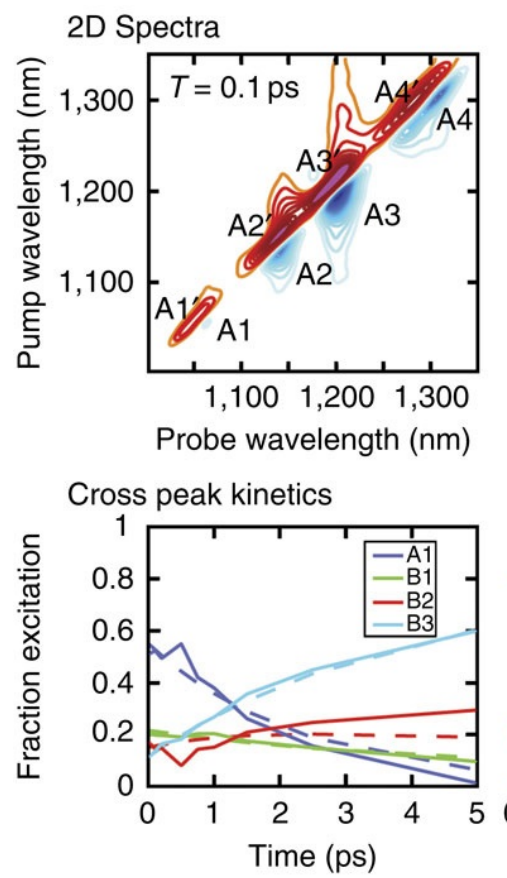

$S_{1} / S_{1}$ quadrant

Submitted to
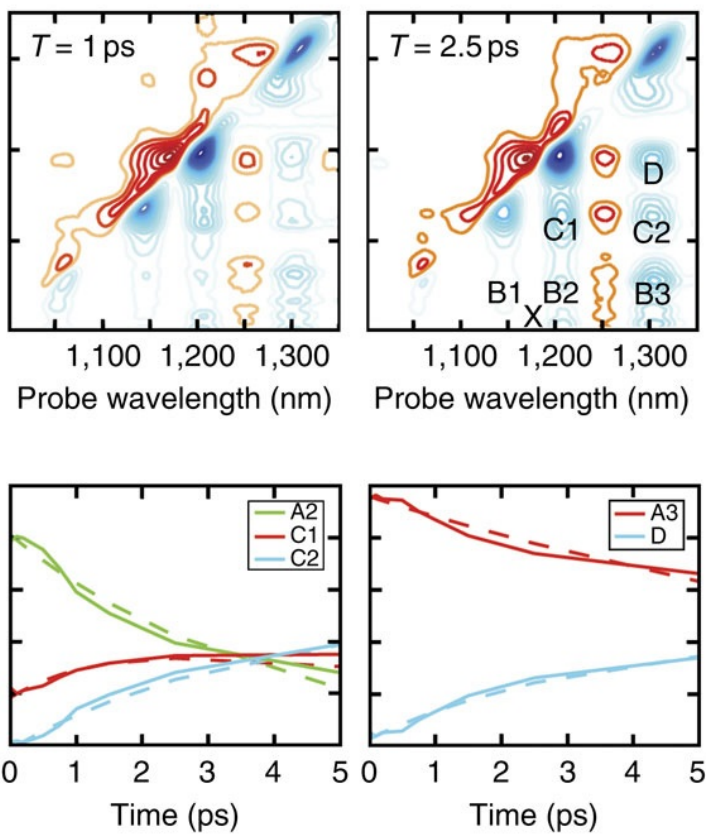

Figure 16. upper panels: 2DES maps for a blend of SWNTs measured for different values of the waiting time T; lower panels: dynamics of the cross peaks indicating energy transfer from the higher to the lower bandgap tubes. Reprinted from [168].

\subsection{Coherent phonons dynamics}

Phonons in SWNTs [169][170] offer distinctive and fundamental physical insights on these quasi ideal 1D systems. Moreover, they represent the basis for the explanation of the non-radiative processes and decay channels that lead to the observed low PL yields in SWNTs. Due to the excitonic character of the optical transitions, strong exciton-phonon coupling is expected [171] and the transfer of the oscillator strength from the excitonic transition to a phonon side-band can be observed in photoluminescence excitation experiments [172] as well as ground state absorption spectra. Raman spectroscopy represents a non-invasive and extremely powerful tool for SWNTs characterization,[173] in particular for chirality [174][175] and defects assignment.[176] The Raman response of SWNTs is dominated by two modes: the radial breathing mode $(\mathrm{RBM})$ and the $\mathrm{G}(\mathrm{C}=\mathrm{C}$ stretching) mode. The $\mathrm{RBM}$ is associated with periodic expansion and contraction of the tube cross section and has a frequency $\omega_{\mathrm{RB}}$ of the order of $100-300 \mathrm{~cm}^{-1}$; since $\omega_{\mathrm{RBM}} \propto 1 / \mathrm{d}$, where $\mathrm{d}$ is the tube diameter, RBMs represent a powerful tool for chirality assignment and for the study of the so called "family behaviours" in SWNTs.[177][178][179][180] G modes are associated to the longitudinal stretching of the C-C bond and are thus present in many carbon-based materials. 
Submitted to

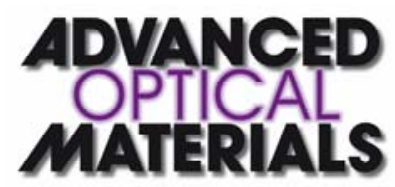

Being characterized by a Raman shift of approximately $1600 \mathrm{~cm}^{-1}$, they are far from Rayleigh scattering and thus easily detectable by resonant Raman experiment.

As explained in section 4.3, ICVS is a powerful technique for time-domain detection of coherent phonons.[178][181] Although it has lower sensitivity with respect to conventional Raman, it enables direct measurement of excited-state vibrational properties [182] and provides intrinsic suppression of the Rayleigh scattering. Lüer et al. [183] measured coherent phonons in a sample of highly pure $(6,5)$ semiconducting SWNTs using sub-10-fs pulses resonant with the $\mathrm{S}_{22}$ transition. Figure 17a shows a $\Delta \mathrm{T} / \mathrm{T}$ map, as a function of probe wavelength and delay. The signal is dominated by the GSB of the $\mathrm{S}_{22}$ transition, which shows an initial ultrafast recovery $\left(\tau \approx 40 \mathrm{fs}\right.$ ) corresponding to the previously discussed $\mathrm{S}_{22} \rightarrow \mathrm{S}_{11}$ relaxation, followed by a slower dynamics corresponding to relaxation from $\mathrm{S}_{11}$ to the ground state. Figure 17b shows the $\Delta \mathrm{T} / \mathrm{T}$ map after subtraction of the population dynamics, which clearly displays an oscillatory pattern due to impulsive excitation of the vibrational coherence in the SWNTs. A Fourier transform (FT) of the oscillations allows to identify two modes: the $\mathrm{RBM}$ at $307 \mathrm{~cm}^{-1}$ and the $\mathrm{G}$ mode at $1596 \mathrm{~cm}^{-1}$. Figure $17 \mathbf{c}$ and $\mathbf{e}$ show the probe wavelength dependence of amplitude and phase of the oscillations, obtained from the FT of the oscillatory component of the signal, for the RBM and the $\mathrm{G}$ mode respectively. For both modes, one observes a minimum of oscillation amplitude at $570 \mathrm{~nm}$, corresponding the peak of the $\mathrm{S}_{22}$ transition, which is associated to a phase jump, which is nearly $\pi$ for the RBM and somewhat less for the $G$ mode. To qualitatively understand this dependence, we recall that impulsive excitation creates a vibrational wave packet, which propagates on the PES according to a quasi-classical sinusoidal trajectory. This wave packet modulates the transition energy of the SWNTs, which periodically shifts to the red or to the blue. Therefore the amplitude profile of the oscillations should resemble the first derivative of the ground state absorption spectrum, while oscillations on opposite sides should display a $\pi$ phase shift. A more rigorous calculation of the amplitude and phase profiles can be obtained by time-dependent wave packet theory under the assumption of a two-level molecular excitonic system.[184] The results of this model are displayed in Figure 17d and $\mathbf{f}$ respectively and are in very good agreement with experimental data. These measurements enable a quantitative determination of electron-phonon coupling in SWNTs, expressed in terms of dimensionless excited-state displacement $\Delta:$ the results are $\Delta_{R B M} \approx 0.15 \div 0.3$ and $\Delta_{G} \approx 0.9 \pm 0.2$ for the $\mathrm{RBM}$ and $\mathrm{G}$ modes respectively. 

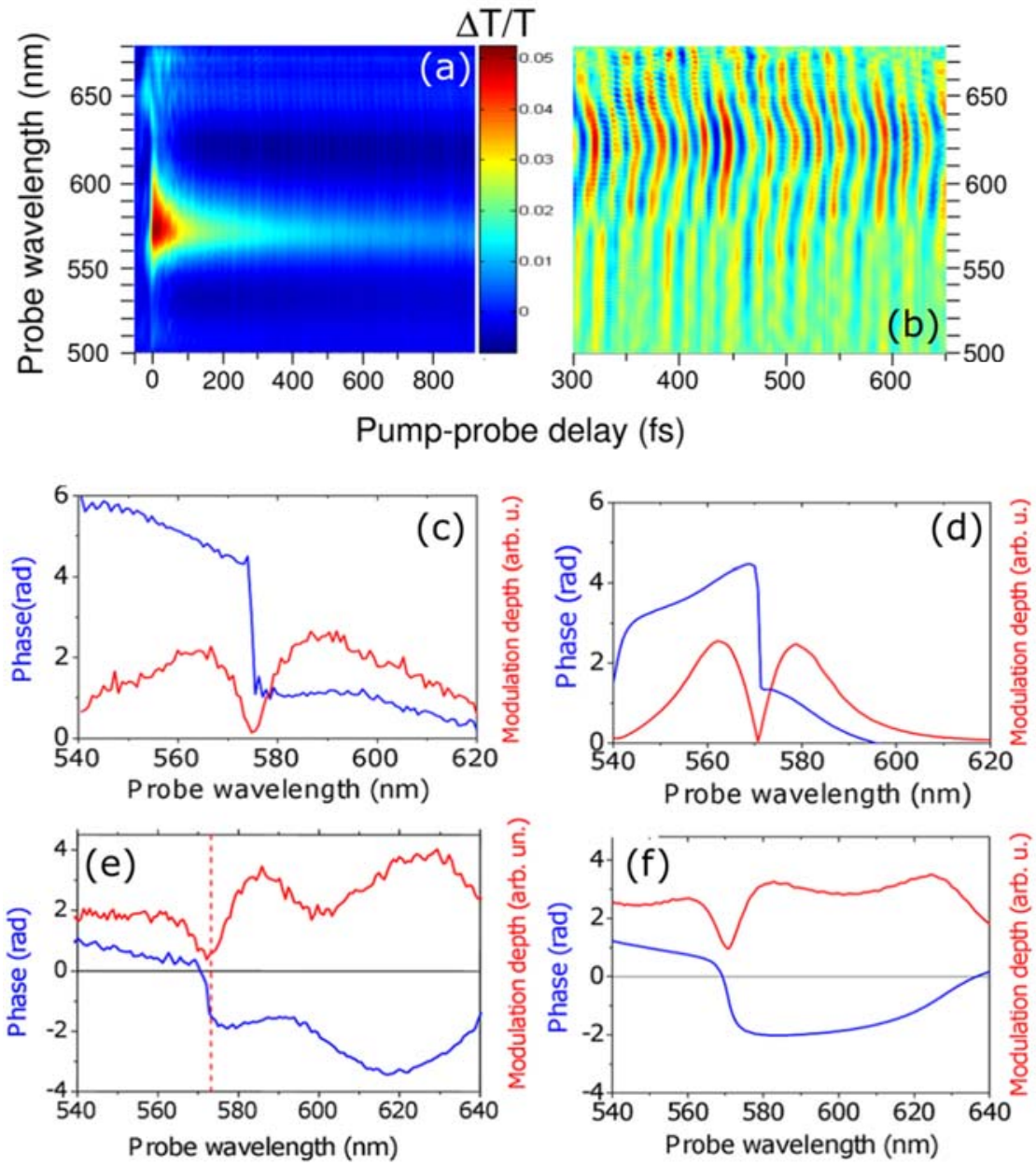

Figure 17. (a-b) $\Delta \mathrm{T} / \mathrm{T}$ map as a function of probe wavelength and pump-probe delay for the $(6,5)$ SWNT excited in resonance with the $S_{22}$ transition. Experimental amplitude (red) and phase (blue) profiles of the (c) RBM and (e) G-mode coherent oscillations. The corresponding simulated profiles are (d) for the RBM and (f) for the G mode. Adapted from [183].

Time-domain ICVS enables additional insight into vibrational dynamics with respect to frequency-domain Raman. Gambetta et al. [185] demonstrated that RBMs and G-modes in SWNTS are anharmonically coupled, resulting in a frequency modulation of the G mode by the RBM. Experiments were performed on SWNTs grown by the high pressure carbon monoxide technique and excited on the $\mathrm{S}_{22}$ transition by sub-10-fs pulses. Figure 18a shows the $\Delta \mathrm{T} / \mathrm{T}$ dynamics at $2.1 \mathrm{eV}$ probe photon energy: besides the usual fast decay, coherent oscillations are observed both for the RBM modes and the $\mathrm{G}$ modes (see inset). Further insight into the vibrational dynamics can be obtained by analysing the data via the sliding window FT (SWFT) which is a series of FTs carried out on different time windows with position swept along the whole time axis of the measurement. The SWFT allows to retrieve 
instantaneous frequencies and their time dynamics. Figure 18b shows the SWFT for the G mode, which indicates a temporal modulation of the G-mode frequency. The period of the modulation, which is extracted by a FT of the time-dependent band peak frequency, strikingly coincides with the period of the RBM (132 fs), indicating that the two modes are nonlinearly coupled. The experimental data can be excellently reproduced by assuming that the G-mode frequency is sinusoidally modulated by the RBM configurational coordinate $\mathrm{Q}_{\mathrm{RBM}}$, i.e. $\omega_{G}=$ $\omega_{G}\left(Q_{R B M}(t)\right)$. Quantum-chemical modelling [186] shows that this effect is due to a corrugation of the SWNT surface on photoexcitation, leading to a coupling between longitudinal and radial vibrations which is detected in the time domain. These results show the unique power of time-domain approaches for the study of vibrational dynamics.
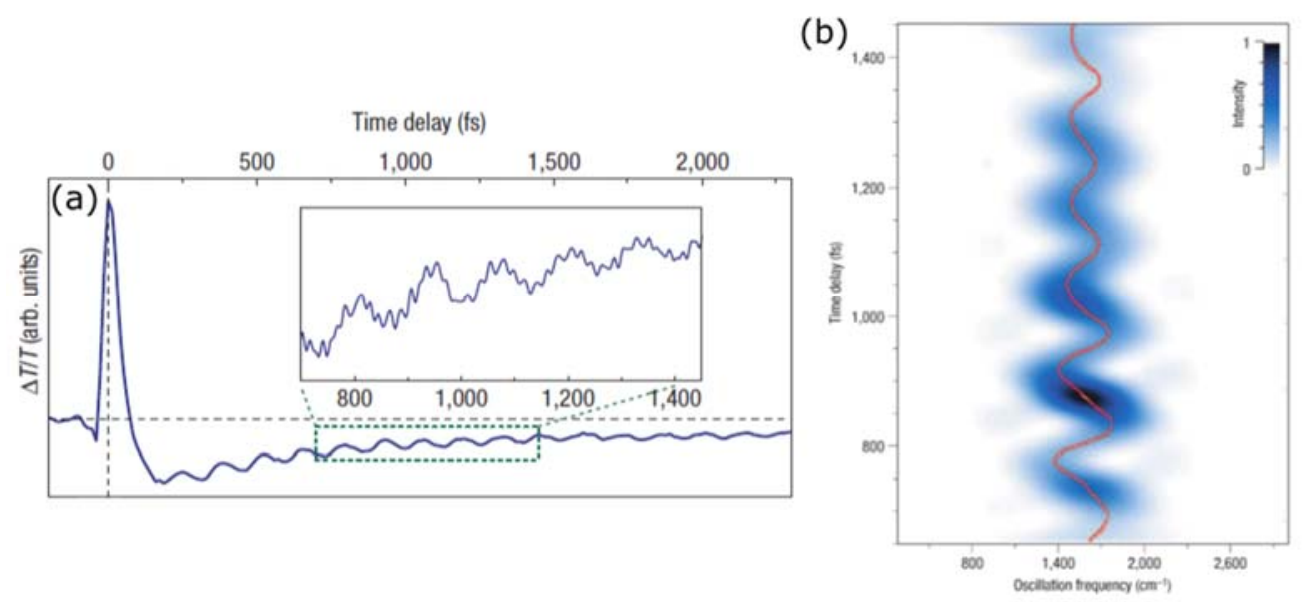

Figure 18. Differential transmission dynamics of SWNTs. a) Excitation and probing by a sub-10-fs visible pulse and detection at $2.1 \mathrm{eV}$. The inset shows a portion of the trace acquired with a higher sampling rate, to fully resolve the high-frequency G-mode oscillations. b) Sliding window Fourier Transform of the data in the inset of (a) shows that the G mode is modulated at the frequency of the RBMs. Adapted from Reference [185].

\section{Conclusions and perspectives}

Over the two decades since their discovery, there has been an impressive progress both in the synthesis and characterization techniques of SWNTs and in the understanding of their basic electronic and optical properties. Thanks to advanced sorting techniques, in particular DGU, it is now possible to produce samples with nearly $100 \%$ chirality selectivity, which has allowed to greatly advance spectroscopic studies. A variety of ultrafast optical spectroscopy techniques has been applied to SWNTs, revealing a rather complex scenario. The primary photoexcitations in SWNTs are singlet excitons, with large binding energy of $\sim 0.5 \mathrm{eV}$ and size of $\sim 2 \mathrm{~nm}$. The higher energy exciton relax to the lowest energy transition $\mathrm{S}_{11}$ on the sub- 
Submitted to

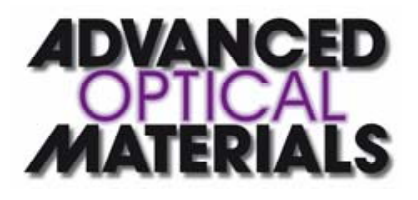

100 -fs timescale. The $\mathrm{S}_{11}$ state, in turn, decays to the ground state on the $\sim 10-100 \mathrm{ps}$ timescale mainly by non-radiative processes, such as trapping to impurities or dark states; this comparatively fast decay is responsible for the very low PL quantum yield of SWNTS. Besides singlet excitons, other species such as triplet excitons, bi-excitons and trions have been identified and manifest themselves by their characteristic transient absorption bands. Finally, the photogeneration of free charges has also been demonstrated, although it remains debated whether they are directly generated or derive from linear or nonlinear exciton dissociation. Excitonic transitions are strongly coupled to phonons, in particular the RBM and the G mode, which can be impulsively excited and detected in the time domain and which have been shown to be nonlinearly coupled.

Despite the impressive available body of work on ultrafast spectroscopy of SWNTs, not all questions have been answered, and further studies will benefit from advances both in synthesis methods and in spectroscopic techniques. In addition, it will be interesting to draw analogies between SWNTs and other quantum confined semiconductors, such as GNRs, which can be nowadays fabricated with $100 \%$ selectivity through bottom-up chemical synthesis. 


\section{Acknowledgements}

\section{ADVANCED \\ MATERIALS}

GC acknowledges support by the EC under Graphene Flagship (contract no. CNECT-ICT604391).

The table of contents entry should be fifty to sixty words long

The interest for Carbon Nanotubes stands at the edge between fundamental physics in quantum confined systems and advanced technology. This review summarizes more than two decades of intense research on their ultrafast photophysical properties, the most advanced spectroscopic techniques used for their optical characterization and possible applications in the fields of photonics, opto-electronics and photovoltaics.

\section{Giancarlo Soavi, Francesco Scotognella, Guglielmo Lanzani and Giulio Cerullo}

\section{Ultrafast Photophysics of Carbon Nanotubes}

\section{ToC figure}

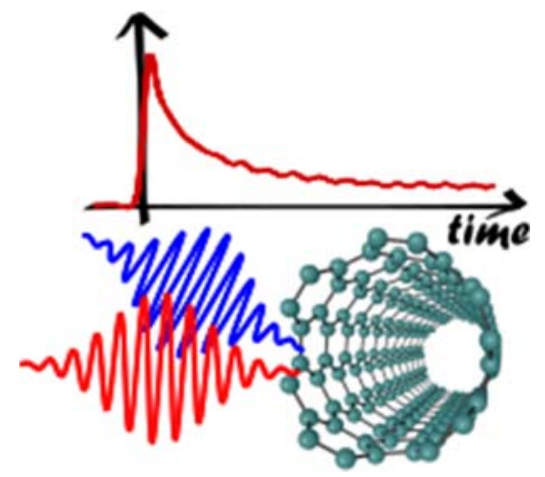




\section{References}

[1] M. Monthioux, V. L. Kuznetsov, Carbon 2006, 44, 1621.

[2] L. V. Radushkevich, V. M. Lukyanovich, Sov. J. Chem. Phys. 1952, $26,88$.

[3] A. Oberlin, M. Endo, T. Koyama, Carbon 1976, 14, 133.

[4] S. Iijima, Nature 1991, 354, 56.

[5] S. Park, M. Vosguerichian, Z. Bao, Nanoscale 2013 5, 1727.

[6] J. M. Schnorr, T. M. Swager, Chem. Mater. 2011, 23, 646.

[7] R. H. Baughman, A. A. Zakhidov, W. A. de Heer, Science 2002, 297, 787.

[8] M. F. De Volder, S. H. Tawfick, R. H. Baughman, A. J. Hart, Science 2013, 339, 535.

[9] C. M. Voge, J. P. Stegemann, J. Neural Eng. 2011, 8, 011001.

[10] M. S. Dresselhaus, G. Dresselhaus, P. C. Eklund, Science of fullerenes and carbon nanotubes: their properties and applications, Academic Press 1996.

[11] P. J. F. Harris, Carbon nanotube science: synthesis, properties and applications, Cambridge University Press 2009.

[12] C. Tan, K. Tan, Y. Ong, A. Mohamed, S. Zein, S. Tan, Environ. Chem. Lett. 2012 10, 265.

[13] S. Lebedkina, P. Schweissb, B. Renkerb, S. Malika, F. Hennrichc, M. Neumaierc, C. Stoermerc, M. M. Kappes, Carbon 2002, 40, 417.

[14] C.D. Scott, S. Arepalli, P. Nikolaev, R.E. Smalley, App. Phys. A 2001, 72, 573.

[15] K. Hata, D. N. Futaba, K. Mizuno, T. Namai, M. Yumura, S. Iijima, Science 2004, 306, 1362.

[16] F. Yang, X. Wang, D. Zhang, J. Yang, D. Luo, Z. Xu, J. Wei, J.-Q. Wang, Z. Xu, F. Peng, X. Li, R. Li, Y. Li, M. Li, X. Bai, F. Ding, Y. Li, Nature 2014, 510, 522.

[17] H. Omachi, T. Nakayama, E. Takahashi, Y. Segawa, K. Itami, Nat. Chem. 2013, 5, 572.

[18] V. C. Moore, M. S. Strano, E. H. Haroz, R. H. Hauge, R. E. Smalley, J. Schmidt, Y. Talmon, Nano Lett. 2003, 1379, 3.

[19] K. D. Ausman, R. Piner, O. Lourie, R. S. Ruoff, M. Korobov, J. Phys. Chem. B 2000, $104,8911$.

[20] M. Zheng, A. Jagota, M. S. Strano, A. P. Santos, P. Barone, S. G. Chou, B. A. Diner, M. S. Dresselhaus, R. S. Mclean, G. B. Onoa, G. G. Samsonidze, E. D. Semke, M. Usrey, D. J. Walls, Science 2003, 302, 1545.

[21] R. Krupke, F. Hennrich, H. v. Löhneysen, M. M. Kappes, Science 2003, 301, 344.

[22] M. S. Arnold, S. I. Stupp, M. C. Hersam, Nano Lett. 2005, 5, 713.

[23] S. A. Hodge, S. Fogden, C. A. Howard, N. T. Skipper, M. S. P. Shaffer, ACS Nano 2013, 7, 1769.

[24] S. A. Hodge, M. K. Bayazit, K. S. Coleman, M. S. P. Shaffer, Chem. Soc. Rev. 2012, 41, 4409.

[25] B. Q. Wei, R. Vajtai, P. M. Ajayan, App. Phys. Lett. 2001, 79, 1172.

[26] B. Peng, M. Locascio, P Zapol, S. Li, S. L. Mielke, G. C. Schatz, H. D. Espinosa, Nat. Nanotech. 2008, 3626.

[27] E. Pop, M. David, W. Qian, G. Kenneth, D. Hongjie, Nano Lett. 2006, 6, 96.

[28] A. E. Aliev, J. Oh, M. E. Kozlov, A. A. Kuznetsov, S. Fang, A. F. Fonseca, R. Ovalle, M. D. Lima, M. H. Haque, Y. N. Gartstein, M. Zhang, A. A. Zakhidov, R. H. Baughman, Science 2009, 323, 1575.

[29] M. D. Lima, N. Li, M. J. de Andrade, S. Fang, J. Oh, G. M. Spinks, M. E. Kozlov, C. S. Haines, D. Suh, J. Foroughi, S. J. Kim, Y. Chen, T. Ware, M. K. Shin, L. D. Machado, A. F. Fonseca, J. D. W. Madden, W. E. Voit, D. S. Galvão, R. H. Baughman, Science 2012, 338, 928.

[30] Q. Cao, J. A. Rogers, Adv. Mater. 2009, 21, 29. 


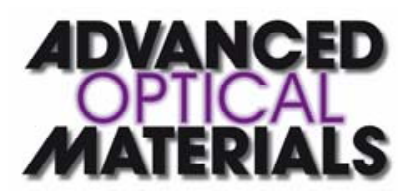

[31] X. Zhou, J.-Y. Park, S. Huang, J. Liu, P. L. McEuen, Phys. Rev. Lett. 2005, 95, 146805.

[32] S. Park, G. Wang, B. Cho, Y. Kim, S. Song, Y. Ji, M.-H. Yoon, T. Lee, Nat. Nanotech. 2012, 7, 438.

[33] A. Maeda, S. Matsumoto, H. Kishida, T. Takenobu, Y. Iwasa, M. Shiraishi, M. Ata, H. Okamoto, Phys. Rev. Lett. 2005, 94, 047404.

[34] T. Hertel, R. Fasel, G. Moos, App. Phys. A 2002, 75, 449.

[35] C. Manzoni, A. Gambetta, E. Menna, M. Meneghetti, G. Lanzani, G. Cerullo, Phys. Rev. Lett. 2005, 94, 207401.

[36] I. H. Baek, S. Y. Choi, H. W. Lee, W. B. Cho, V. Petrov, A. Agnesi, V. Pasiskevicius, D.-I. Yeom, K. Kim, F. Rotermund, Opt. Exp. 2011, 8, 7833.

[37] F. Wang, A. G. Rozhin, V. Scardaci, Z. Sun, F. Hennrich, I. H. White, W. I. Milne, A. C. Ferrari, Nat. Nanotech. 2008, 3, 738.

[38] P. Avouris, M. Freitag, V. Perebeinos, Nat. Photon. 2008, 2, 341.

[39] Y. Liu, F. Wang, X. Wang, X. Wang, E. Flahaut, X. Liu, Y. Li, X. Wang, Y. Xu, Y. Shi, R. Zhang, Nat Comms 2015, 6, 8589.

[40] M. S. Arnold, J. L. Blackburn, J. J. Crochet, S. K. Doorn, J. G. Duque, A. Mohitee, H. Telge, Phys. Chem. Chem. Phys. 2013, 15, 14896.

[41] M. Barkelid, V. Zwiller, Nat. Photon. 2014, 8, 7.

[42] D. J. Bindl, M. S. Arnold, J. Phys. Chem. C 2013, 117, 2390.

[43] D. J. Bindl, M.-Y. Wu, F. C. Prehn, M. S. Arnold, Nano Lett. 2011, 11, 455.

[44] J.-C. Charlier, X. Blase, S. Roche, Rev. Mod. Phys. 2007, 79, 677.

[45] P. R. Wallace, Phys. Rev. 1947, 71, 622.

[46] J. C. Slonczewski, P. R. Weiss, Phys. Rev. 1958, 109, 272.

[47] A. H. Castro Neto, F. Guinea, N. M. R. Peres, K. S. Novoselov, A. K. Geim, Rev. Mod. Phys. 2009, 81, 109.

[48] X. Blase, L. X. Benedict, E. L Shirley, S. G Louie, Phys. Rev. Lett. 1994, 72, 1878.

[49] M. S. Arnold, A. A. Green, J. F. Hulvat, S. I. Stupp, M. C. Hersam, Nat. Nanotechnol. 2006, 1,60 .

[50] J. Crochet, M. Clemens, T. Hertel, J. Am. Chem. Soc. 2007, 1298058.

[51] P. Delaney, H. J. Choi, J. Ihm, S. G Louie, M. L Cohen, Nature 1998, 391, 466.

[52] P. G. Collins, K. Bradley, M. Ishigami, A. Zettl, Science 2000, 287, 1801.

[53] A. Zahab, L. Spina, P. Poncharal, C. Marliére, Phys. Rev. B 2000, 62, 10000.

[54] L. X. Benedict, S. G. Louie, M. L. Cohen, Phys. Rev. B 1995, 52, 8541.

[55] H. Kataura, Y. Kumazawa, Y. Maniwa, I. Umezu, S. Suzuki, Y. Ohtsuka, Y. Achiba, Synth. Met. 1999, 103, 2555.

[56] C. T. White, J. W. Mintmire, Nature 1998, 394, 29.

[57] R. Saito, G. Dresselhaus, M. S. Dresselhaus, Phys. Rev. B 2000, 61, 2981.

[58] S. Bachilo, M. S. Strano, C. Kittrell, R. H. Hauge, R. E. Smalley,R. B. Weisman, Science 2002, 298, 2361.

[59] M. J. O' Connell, S. M. Bachilo, C. B. Huffman, V. C. Moore, M. S. Strano, E. H. Haroz, K. L. Rialon, P. J. Boul, W. H. Noon, C. Kittrell, J. Ma, R. H. Hauge, R. B. Weisman, R. E. Smalley, Science 2002, 297, 593.

[60] J. W. Mintmire, C. T. White, Phys. Rev. Lett. 1998, 81, 2506.

[61] C. L. Kane, E. J. Mele, Phys. Rev. Lett. 2003, 90, 207401.

[62] R. J. Elliott, Phys. Rev. 1957, 108, 1384.

[63] R. Loudon, Am J. Phys. 1959, 27, 649.

[64] F. Wang, G. Dukovic, L. E. Brus, T. F. Heinz, Science 2005, 308, 838.

[65] J. Maultzsch, R. Pomraenke, S. Reich, E. Chang, D. Prezzi, A. Ruini, E. Molinari, M. S. Strano, C. Thomsen, C. Lienau, Phys. Rev. B 2005 72, 241402. 
[66] T. Ando, J. Phys. Soc. Jpn. 1997, 66, 1066.

Submitted to

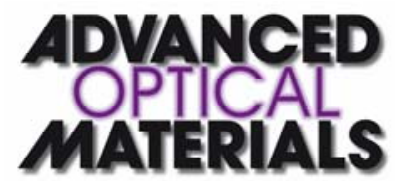

[67] C. D. Spataru, S. Ismail-Beigi, L. X. Benedict, S. G. Louie, Phys. Rev. Lett. 2004, 92, 077402.

[68] V. Perebeinos, J. Tersoff, P. Avouris, Phys. Rev. Lett. 2004, 92, 257402.

[69] T. G. Pedersen, K. Pedersen, H. D. Cornean, P. Duclos, Nano Lett. 2005, 5, 291.

[70] L. Colombier, J. Selles, E. Rousseau, J.-S. Lauret, F. Vialla, C. Voisin, G. Cassabois, Phys. Rev. Lett. 2012, 109, 197402.

[71] T. F. Rønnow, T. G. Pedersen, H. D. Cornean, Phys. Rev. B 2010, 81, 205446.

[72] K. Watanabe, K. Asano, Phys. Rev. B 2012, 85, 035416.

[73] M. C. Beard, J. L. Blackburn, M. J. Heben, Nano Lett. 2008, 8, 4238.

[74] G. Soavi, F. Scotognella, D. Brida, T. Hefner, F. Spaeth, M. R. Antognazza, T. Hertel, G. Lanzani, G. Cerullo, J. Phys. Chem. C 2013, 117, 10849.

[75] S. A. Jensen, R. Ulbricht, A. Narita, X. Feng, K. Muellen, T. Hertel, D. Turchinovich, M. Bonn, Nano Lett. 2013, 13, 5925.

[76] J. Park, O. G. Reid, J. L. Blackburn, G. Rumbles, Nat. Comms. 2015, 6, 8809.

[77] Z. Vardeny, J. Tauc, Opt. Commun. 1981, 39, 396.

[78] S. A. Kovalenko, A. L. Dobryakov, J. Ruthmann, N. P. Ernsting, Phys. Rev. A 1999, 59, 2369.

[79] S. Gélinas, A. Rao, A. Kumar, S. L. Smith, A. W. Chin, J. Clark, T. S. van der Poll, G. C. Bazan, R. H. Friend, Science 2014, 343, 512.

[80] E. A. A. Pogna, M. Marsili, D. De Fazio, S. Dal Conte, C. Manzoni, D. Sangalli, D. Yoon, A. Lombardo, A. C. Ferrari, A. Marini, G. Cerullo, D. Prezzi, ACS Nano 2016, $10,1182$.

[81] T. Elsaesser, W. Kaiser, Annu. Rev. Phys. Chem. 1991, 42, 83.

[82] E. R. Thoen, G. Steinmeyer, P. Langlois, E. P. Ippen, G. E. Tudury, C. H. Brito Cruz, L. C. Barbosa, C. L. Cesar, Appl. Phys. Lett. 1998, 73, 2149.

[83] D. Brida, C. Manzoni, G. Cirmi, M. Marangoni, S. Bonora, P. Villoresi, S. De Silvestri, G. Cerullo, J. Opt. 2009, 12, 013001.

[84] D. Polli, L. Lüer, G. Cerullo, Rev. Sci. Instrum. 2007, 78, 103108.

[85] T. Yajima, Y. Taira, J. Phys. Soc. Jpn. 1979, 47, 1620.

[86] A. M. Weiner, S. De Silvestri, E. P. Ippen, J. Opt. Soc. Am. B 1985, 2, 654.

[87] T. Joo, Y. Jia, J.-Y. Yu, M. J. Lang, G. R. Fleming, J. Chem. Phys. 1996, 104, 6089.

[88] S. Mukamel, Annu. Rev. Phys. Chem. 2000, 51, 691.

[89] T. Brixner, I. V. Stiopkin, G. R. Fleming, Opt. Lett. 2004, $29,884$.

[90] E. M. Grumstrup, S. H. Shim, M. A. Montgomery, N. H. Damrauer, M. T. Zanni, Opt. Express 2007, 15, 16681.

[91] T. Zhang, C. N. Borca, X. Li, S. T. Cundiff, Opt. Exp. 2005, 13, 7432.

[92] J. Helbing, P. Hamm, J. Opt. Soc. Am. B 2011, 28, 171.

[93] D. B. Turner, K. W. Stone, K. Gundogdu, K. A. Nelson, Rev. Sci. Instrum. 2011, 82, 081301.

[94] J. A. Myers, K. L. Lewis, P. F. Tekavec, J. P. Ogilvie, Opt. Exp. 2008, 16, 17420.

[95] D. Brida, C. Manzoni, G. Cerullo, Opt. Lett. 2012, 37, 3027.

[96] M. Liebel, C. Schnedermann, T. Wende, and P. Kukura, J. Phys. Chem. A 2015, 119, 9506.

[97] G. Lanzani, G. Cerullo, C. Brabec, N.S. Sariciftci, Phys. Rev. Lett. 2003, 90, 047402.

[98] S. Mukamel, Principles of Nonlinear Optical Spectroscopy, Oxford University Press, USA, 1995.

[99] S. Ruhman, B. Kohler, A. G. Joly, K. A. Nelson, Chem. Phys. Lett. 1987, 141, 16. [100] J. Lefebvre, P. Finnie, Nano Lett. 2008, 8, 1890. 
Submitted to

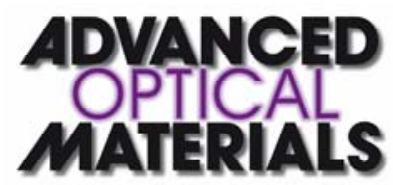

[101] G. Dukovic, F. Wang, D. Song, M. Y. Sfeir, T. F Heinz, L. E. Brus, Nano Lett. 2005, $5,2314$.

[102] J. Shah, Ultrafast Spectroscopy of Semiconductors and Semiconductor Nanostructures, Springer, 1999.

[103] O. J. Korovyanko, C.-X. Sheng, Z. V. Vardeny, A. B. Dalton, R. H. Baughman, Phys. Rev. Lett. 2004, 92, 017403.

[104] Y.-Z. Ma, L. Valkunas, S. M. Bachilo, G. R. Fleming, J. Phys. Chem. B 2005,109, 15671.

[105] Y.-Z. Ma, L. Valkunas, S. L. Dexheimer, S. M. Bachilo, G. R. Fleming, Phys. Rev. Lett. 2005, 94, 157402.

[106] A. Jorio, P. T. Araujo, S. K. Doorn, S. Maruyama, H. Chacham, M. A. Pimenta, phys. status solidi $B$ 2006, 243, 3117.

[107] P. T. Araujo, S. K. Doorn, S. Kilina, S. Tretiak, E. Einarsson, S. Maruyama, H. Chacham, M. A. Pimenta, A. Jorio, Phys. Rev. Lett. 2007, 98, 067401.

[108] T. Michel, M. Paillet, J. C. Meyer, V. N. Popov, L. Henrard, J.-L. Sauvajol, Phys. Rev. B 2007, 75, 155432.

[109] S. Berciaud, C. Voisin, H. Yan, B. Chandra, R. Caldwell, Y. Shan, L. E. Brus, J. Hone, T. F Heinz, Phys. Rev. B 2010, 81, 041414.

[110] G. Soavi, F. Scotognella, D. Viola, T. Hefner, T. Hertel, G. Cerullo, G. Lanzani, Sci. Rep. 2015, 5, 9681.

[111] S. Tretiak, S. Kilina, A. Piryatinski, A. Saxena, R. L. Martin, A. R Bishop, Nano Lett. 2007, 7, 86.

[112] L. Lüer, S. Hoseinkhani, D. Polli, J. Crochet, T. Hertel, G. Lanzani, Nat. Phys. 2008, 5,54 .

[113] B. I. Greene, J. Orenstein, S. Schmitt-Rink, Science 1990, 247, 679.

[114] L. Cognet, D. A. Tsyboulski, J.-D. R. Rocha, C. D. Doyle, J. M. Tour, R. B. Weisman, Science 2007, 316, 1465.

[115] T. Hertel, S. Himmelein, T. Ackermann, D. Stich, J. Crochet, ACS Nano 2010, 4, 7161.

[116] Y.-Z. Ma, M. W. Graham, G. R. Fleming, A. A. Green, M. C. Hersam, Phys. Rev. Lett. 2008, 101, 217402.

[117] M. W. Graham, Y.-Z. Ma, G. R. Fleming, Nano Lett. 2008, 8, 3936.

[118] M. W. Graham, Y.-Z. Ma, A. A. Green, M. C. Hersam, G. R. Fleming, J. Chem. Phys. 2011, 134, 034504.

[119] J. R. Schneck, A. G. Walsh, A. A. Green, M. C. Hersam, L. D. Ziegler, A. K. Swan, J. Phys. Chem. A 2011, 115, 3917.

[120] M. W. Graham, T. R. Calhoun, A. A. Green, M. C. Hersam, G. R. Fleming, Nano Lett. 2012, 12, 813.

[121] B. Yuma, S. Berciaud, J. Besbas, J. Shaver, S. Santos, S. Ghosh, R. B. Weisman, L. Cognet, M. Gallart, M. Ziegler, B. Hönerlage, B. Lounis, P. Gilliot, Phys. Rev. B 2013, $87,205412$.

[122] T. Hertel, A. Hagen, V. Talalaev, K. Arnold, F. Hennrich, M. Kappes, S. Rosenthal, J. McBride, H. Ulbricht, E. Flahaut, Nano Lett. 2005, 5, 511.

[123] J.-S. Lauret, C. Voisin, G. Cassabois, C. Delalande, P. Roussignol, O. Jost, L. Capes, Phys. Rev. Lett. 2003, 90, 057404.

[124] Y.-Z. Ma, J. Stenger, J. Zimmermann, S. M. Bachilo, R. E. Smalley, R. B. Weisman, G. R. Fleming, J. Chem. Phys. 2004, 120, 3368.

[125] L. Lüer, G. Lanzani, J. Crochet, T. Hertel, J. Holt, Z. V. Vardeny, Phys. Rev. B 2009, 80, 205411. 


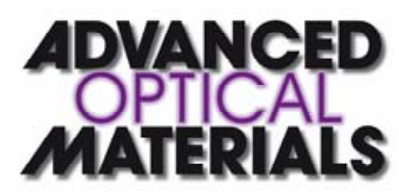

[126] N. Akizuki, S. Aota, S. Mouri, K. Matsuda,. Y. Miyauchi, Nat. Comm. 2015, 6, 8920.

[127] F. Wang, G. Dukovic, L. E. Brus, T. F. Heinz, Phys. Rev. Lett. 2004, 92, 177401.

[128] S. Lebedkin, F. Hennrich, T. Skipa, M. M. Kappes, J. Phys. Chem. B 2003, 107, 1949.

[129] D. J. Styers-Barnett, S. P. Ellison, C. Park, K. E. Wise, J. M. Papanikolas, J. Phys. Chem. A 2005, 109, 289.

[130] S. G. Chou, M. F. DeCamp, J. Jiang, G.G. Samsonidze, E. B. Barros, F. Plentz, A. Jorio, M. Zheng, G. B. Onoa, E. D. Semke, A. Tokmakoff, R. Saito, G. Dresselhaus, M. S. Dresselhaus, Phys. Rev. B 2005, 72, 195415.

[131] L. Valkunas, Y.-Z. Ma, G. R. Fleming, Phys. Rev. B 2006, 73, 115432.

[132] L. Huang, T. D. Krauss, Phys. Rev. Lett. 2006, 96, 057407.

[133] H. van Amerongen, L. Valkunas, R. van Grondelle, Photosynthetic Excitons, World Scientific, Singapore, 2000.

[134] S. Wang, M. Khafizov, X. Tu, M. Zheng, T. D. Krauss, Nano Lett. 2010, 10, 2381.

[135] C. Smith, D. Binks, Nanomaterials 2014, 4, 19.

[136] G. Soavi, S. Dal Conte, C. Manzoni, D. Viola, A. Narita, Y. Hu, X. Feng, U.

Hohenester, E. Molinari, D. Prezzi, K. Müllen, G. Cerullo, Nat. Comm. 2016, 7, 11010.

[137] O. A. Dyatlova, C. Köhler, E. Malic, J. Gomis-Bresco, J. Maultzsch, A. Tsagan-

Mandzhiev, T. Watermann, A. Knorr, U. Woggon, Nano Lett. 2012, 12, 2249.

[138] J. Kono, G. N. Ostojic, S. Zaric, M. S. Strano, V. C. Moore, J. Shaver, R. H. Hauge, R. E. Smalley, Appl. Phys. A 2004, 78, 1093.

[139] A. Hagen, M. Steiner, M. B. Raschke, C. Lienau, T. Hertel, H. Qian, A. J. Meixner, A. Hartschuh, Phys. Rev. Lett. 2005, 95, 197401.

[140] S. Berciaud, L. Cognet, B. Lounis, Phys. Rev. Lett. 2008, 101, 077402.

[141] G. Soavi, A. Grupp, A. Budweg, F. Scotognella, T. Hefner, T. Hertel, G. Lanzani, A. Leitenstorfer, G. Cerullo, D. Brida, Nanoscale 2015, 7, 18337.

[142] I. V. Zozulenko, Solid State Commun. 1990, 76, 1035.

[143] D. Stich, F. Späth, H. Kraus, A. Sperlich, V. Dyakonov, T. Hertel, Nat. Photon. 2014, 8, 139.

[144] R. M. Russo, E. J. Mele, C. L. Kane, I. V. Rubtsov, M. J. Therien, D. E. Luzzi, Phys. Rev. B 2006, 74, 041405(R).

[145] J. Allam, M. T. Sajjad, R. Sutton, K. Litvinenko, Z. Wang, S. Siddique, Q.-H. Yang, W. H. Loh, T. Brown, Phys. Rev. Lett. 2013, 111, 197401.

[146] D. J. Styers-Barnett, S. P. Ellison, B. P. Mehl, B. C. Westlake, R. L. House, C. Park, K. E. Wise, J. M. Papanikolas, J. Phys. Chem C 2008, 112, 4507.

[147] B. Gao, G. V. Hartland, L. Huang, ACS Nano 2012, 6, 5083.

[148] J. Park, P. Deria, M. J. Therien, J. Am. Chem. Soc. 2011, 133, 17156.

[149] S. Tretiak, Nano Lett. 2007, 7, 2201.

[150] S. M. Santos, B. Yuma, S. Berciaud, J. Shaver, M. Gallart, P. Gilliot, L. Cognet, B. Lounis, Phys. Rev. Lett. 2011, 107, 187401.

[151] D. Kammerlander, D. Prezzi, G. Goldoni, E. Molinari, U. Hohenester, Phys. Rev. Lett. 2007, 99, 126806.

[152] B. Gao, G. V. Hartland, L. Huang, J. Phys. Chem. Lett. 2013, 4, 3050.

[153] G. N. Ostojic, S. Zaric, J. Kono, V. C. Moore, R. H. Hauge, R. E. Smalley, Phys. Rev. Lett. 2005, 94, 097401.

[154] J. J. Crochet, S. Hoseinkhani, L. Lüer, T. Hertel, S. K. Doorn, G. Lanzani, Phys. Rev. Lett. 2011, 107, 257402.

[155] T. Koyama, S. Yoshimitsu, Y. Miyata, H. Shinohara, H. Kishida, A. Nakamura, J. Phys. Chem. C 2013, 117, 20289. 
Submitted to

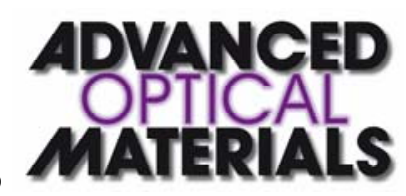

[156] C. Sciascia, J. J. Crochet, T. Hertel, G. Lanzani, Eur. Phys. J. B 2010, 75, 115.

[157] J. Deslippe, C. D. Spataru, D. Prendergast, S. G. Louie, Nano Lett. 2007, 7, 1626.

[158] C. Gadermaier, E. Menna, M. Meneghetti, W. J. Kennedy, Z. V. Vardeny, G. Lanzani, Nano Lett. 2006, 6, 301.

[159] Y. Kumamoto, M. Yoshida, A. Ishii, A. Yokoyama, T. Shimada, Y. K. Kato, Phys. Rev. Lett. 2014, 112, 117401.

[160] V. Perebeinos, P. Avouris, Nano Lett. 2007, 7, 609.

[161] A. D. Mohite, J.-T. Lin, G. Sumanasekera, B. W. Alphenaar, Nano Lett. 2006, 6, 1369.

[162] A. D. Mohite, P. Gopinath, H. M. Shah, B. W. Alphenaar, Nano Lett. 2008, 8, 142.

[163] D. J. Bindl, A. J. Ferguson, M.-Y. Wu, N. Kopidakis, J. L. Blackburn, M. S. Arnold, J. Phys. Chem. Lett. 2013, 4, 3550.

[164] A. Malapanis, V. Perebeinos, D. P. Sinha, E. Comfort, J. U. Lee, Nano Lett. 2013, 13,3531 .

[165] L. Lüer, J. J. Crochet, T. Hertel, G. Cerullo, G. Lanzani, ACS Nano 2010, 4, 4265.

[166] R. D. Mehlenbacher, M.-Y. Wu, M. Grechko, J. E. Laaser, M. S. Arnold, M. T. Zanni, Nano Lett. 2013, 13, 1495.

[167] F. Jakubka, C. Backes, F. Gannott, U. Mundloch, F. Hauke, A. Hirsch, J. Zaumseil, ACS Nano 2013, 7, 7428.

[168] R. D. Mehlenbacher, T. J. McDonough, M. Grechko, M.-Y. Wu, M. S. Arnold, M.

T. Zanni, Nat. Comm. 2015, 6, 6732.

[169] M. S. Dresselhaus, P. C. Eklund, Adv. Phys. 2000, 49, 705.

[170] P. C. Eklund, J. M. Holden, R. A. Jishi, Carbon 1995, 33, 959.

[171] V. Perebeinos, J. Tersoff, P. Avouris, Phys. Rev. Lett. 2005, 94, 027402.

[172] F. Plentz, H. B. Ribeiro, A. Jorio, M. S. Strano, M. A. Pimenta, Phys. Rev. Lett. 2005, 95, 247401.

[173] A. Jorio, M. A. Pimenta, A. G. Souza Filho, R. Saito, G. Dresselhaus, M. S. Dresselhaus, New. J. Phys. 2003, 5, 139.

[174] C. Thomsen, S. Reich, J. Maultzsch, Philos. Trans. R. Soc. A 2004, 362, 2337.

[175] J. Maultzsch, H. Telg, S. Reich, C. Thomsen, Phys. Rev. B 2005, 72, 205438.

[176] M. A. Pimenta, A. Jorio, S. D. M. Brown, A. G. Souza Filho, G. Dresselhaus, J. H. Hafner, C. M. Lieber, R. Saito, M. S. Dresselhaus, Phys. Rev. B 2001, 64, 041401.

[177] I. Eom, S. Park, H.-S. Han, K.-J. Yee, S.-H. Baik, D.-Y. Jeong, T. Joo, Y.-S. Lim, Nano Lett. 2012, 12, 769.

[178] J.-H. Kim, K.-J. Han, N.-J. Kim, K.-J. Yee, Y.-S. Lim, G. D. Sanders, C. J. Stanton, L. G. Booshehri, E. H. Hároz, J. Kono, Phys. Rev. Lett. 2009, 102, 037402.

[179] G. D. Sanders,C. J. Stanton, J.-H. Kim, K.-J. Yee, Y.-S. Lim, E. H. Hároz, L. G. Booshehri, J. Kono, R. Saito, Phys. Rev. Lett. 2009, 79, 205434.

[180] Y.-S. Lim, J.-G. Ahn, J.-H. Kim, K.-J. Yee, T. Joo, S.-H. Baik, E. H. Hároz, L. G. Booshehri, J. Kono, ACS Nano 2010, 4, 3222.

[181] J.-H. Kim, K.-J. Yee, Y.-S. Lim, L. G. Booshehri, E. H. Hároz, J. Kono, Phys. Rev $B$ 2012, 86, 161415(R).

[182] W. T. Pollard, S.-Y. Lee, R. A. Mathies, J. Chem. Phys. 1990, 92, 4012.

[183] L. Lüer, C. Gadermaier, J. Crochet, T. Hertel, D. Brida, G. Lanzani, Phys. Rev. Lett. 2009, 102, 127401.

[184] A. T. N. Kumar, F. Rosca, P. M. Champion, J. Chem. Phys. 2001, 114, 701.

[185] A. Gambetta, C. Manzoni, E. Menna, M. Meneghetti, G. Cerullo, G. Lanzani, S.

Tretiak, A. Piryatinski, A. Saxena, R. L. Martin, A. R. Bishop, Nat Phys. 2006, 2, 515.

[186] S. Tretiak, A. Saxena, R. L. Martin, A. R. Bishop, Phys. Rev. Lett. 2002, 89, 097402. 